\title{
Feature lines modification based on As-Stiff-As-Needed surface deformation
}

\author{
Y. Le Vaou ${ }^{1,2}$, J.-C. Léon*1, S. Hahmann ${ }^{\dagger 1}$, S. Masfrand ${ }^{2}$, and M. Mika² \\ ${ }^{1}$ Univ. Grenoble Alpes, Grenoble INP, CNRS, Inria, LJK, 38000 Grenoble, France \\ ${ }^{2}$ Groupe PSA, Centre Technique de Vélizy, 78140 Vélizy-Villacoublay, France
}

\begin{abstract}
Aesthetic surface mesh modification, guided by a deforming feature line, is still a challenging task. Whereas most existing surface deformation methods work well when the new surface is stretched or isometrically deformed, in case of areas subjected to compression, unwanted bulging may appear. This configuration typically appears when a designer slides a feature line along the input surface. In this paper, we present a new approach for feature line modification solving the surface bulging problem. We consider physically-based deformation methods applied to surface meshes and characterize configurations that produce compression loading from a mechanical standpoint. Then, the deformation area is decomposed into two sub-domains with a boundary modification of the one subjected to compression loading. Thus, during a subsequent deformation, this sub-domain no longer undergoes a compression loading but rather a traction loading, which ultimately prevents bulging. This transformation looks like a retraction of this surface area and a corresponding processing pipeline is set up. Our feature line modification method is intuitive to use and runs interactively, making it attractive to be used during design reviews in immersive environments. Industrial examples and comparisons with state-of-theart physically-based deformation methods validate the proposed approach.
\end{abstract}

Keywords: feature lines, mesh deformation, physicalbased deformation, aesthetic design.

\footnotetext{
*jean-claude.leon@grenoble-inp.fr

†stefanie.hahmann@inria.fr
}

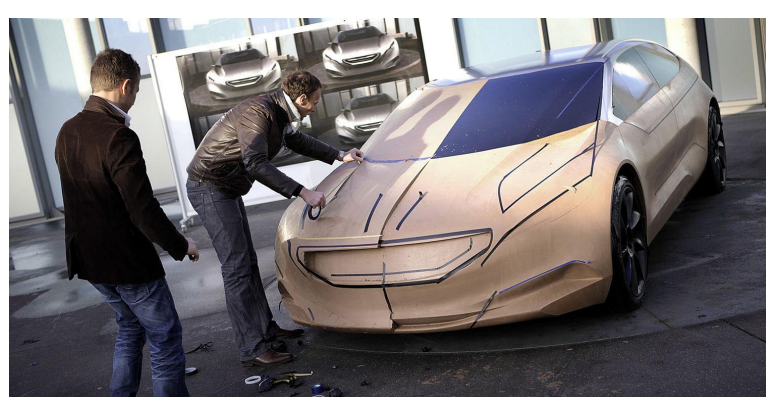

Figure 1: Designers using black tape to visualize the desired feature lines [Courtesy Peugeot Design Lab http://www.peugeotdesignlab.com/fr/ le-studio/concept-cars/peugeot-hxl].

\section{Introduction}

In the automotive industry and at the early design stages of a car body, feature lines $(F L)$ are commonly defined as sharp lines in the surface model of a car body. As FL greatly contribute to the model's aesthetic design, they are often modified during design reviews.

During design reviews around physical mock-ups, FL modifications are defined using a black tape, see Figure 1. The modified lines, here called target lines $(T L)$, thus lie on the initial surface.

Nowadays, virtual reality technologies become part of these design reviews. However, these technologies still lack modification processes, i.e., the ability to mimic this tape-based modification process and deform the underlying surface model. Such a modification process includes, as a whole:

- the generation of the modified FL that can be seen either as a generative process or as a deformation process; 
- the subsequent surfaces deformation;

- the interaction taking place in a virtual environment, that requires taking into account the immersive nature of this environment and the interactive time constraint of the interaction.

Here, the contribution focuses on the generation of the modified surface, considering the initial surface mesh, the FL and the TL as input parameters. We leave the Human-Computer Interaction design, subjected to the interactive time and immersive environment constraints, as objectives for future work. Similarly, if aesthetics is prominent in the surfaces addressed here, the development of an aesthetic criterion to evaluate the deformed surface is not targeted. Rather, the surface bulging phenomenon being characterized by undesired surface undulations, the deformed surfaces are examined through discrete Gaussian curvature sign maps to evaluate the efficiency of the proposed approach.

In order to reduce the amount of input parameters, we focus on physically-based surface mesh deformation methods [46, 8, 9, 30, 53], using the handle metaphor, to best fit the interactive setting of aesthetic design. Conforming to this metaphor, the user selects a Region of Interest (ROI) on the surface, a handle, and applies Euclidean transformations to the handle, regarded as a rigid body, to modify the ROI. Now, a key distinction with our context is that the FL must be deformed to match the TL, and thus cannot be straightforwardly regarded as a handle.

The TL can be positioned anywhere in 3D space, though it often stays close to the initial surface in design reviews, because feature lines aim at bringing character to a model rather than driving the overall shape of an aesthetic model. Consequently, modifications of FL can be divided into two categories:

- modifications "off" the surface: there, every point of the FL moves along a trajectory that is roughly along the average surface normal, stretching the surface on both sides of the FL;

- modifications "on" the surface: every point of the FL follows a trajectory that is stretching the surface on only one side of the FL, depending on the relative location of TL wrt the FL. This configuration is close to the tape modification illustrated in Fig. 1 though the TL may not lie exactly on the initial surface.

Surface mesh modification guided by a deforming FL is still challenging, especially when the FL deforms "on" the surface. In this case, part of the surface is indeed subjected to a compression loading. Whereas most existing methods work well when the deformed surface is stretched or isometrically deformed, in case of compression, unwanted bulging appears with all methods we have tested (see an example in Figure 2).

This unwanted bulging can be seen, from a mechanical standpoint, as a buckling or wrinkling phenomenon, which unfortunately would result in complex optimization problems.

In this paper we solve the bulging problem by presenting a new approach for shape modification driven by FL deformation. Within the scope of physically-based surface mesh deformations, we show that the stiffness distribution brought by the AsStiff-As-Needed (ASAN) surface mesh deformation method [30] is well suited for FL modifications, and propose to carry out a special treatment on the part of the surface subjected to compression. This preprocessing step looks like a retraction of the surface, after which the surface no longer undergoes a compression loading but rather a traction one, which ultimately prevents surface bulging.

The paper is structured as follows. Section 2 reviews surface deformation methods able to process FL modifications. Section 3 details the on/off-surface categorization and gives the overview of the proposed approach for both cases. Then, Section 4 describes the retraction process, applied to a sub-domain to avoid compression loads. Section 5 details the various steps of our deformation method, before showing results and their analysis in Section 6.

\section{Related Work}

The generation of the modified FL can be seen either as a generative process $[21,41,28]$ or a deformation process [18]. We assume that the TL is given as input, so here we review the work to draw smooth lines in an immersive environment in a very brief way.

Drawing 3D curves freehand This is still a challenging task, and the main difficulty seems to be the compromise between intuitiveness [50, 43, 27, 26, 47] and precision $[4,1]$, thus quality, of the line. We note that spline representations of curves are generally preferred in $3 \mathrm{D}$ modeling systems, when a direct control over the shape (curvature and torsion) is required [50, 17, 24]. 

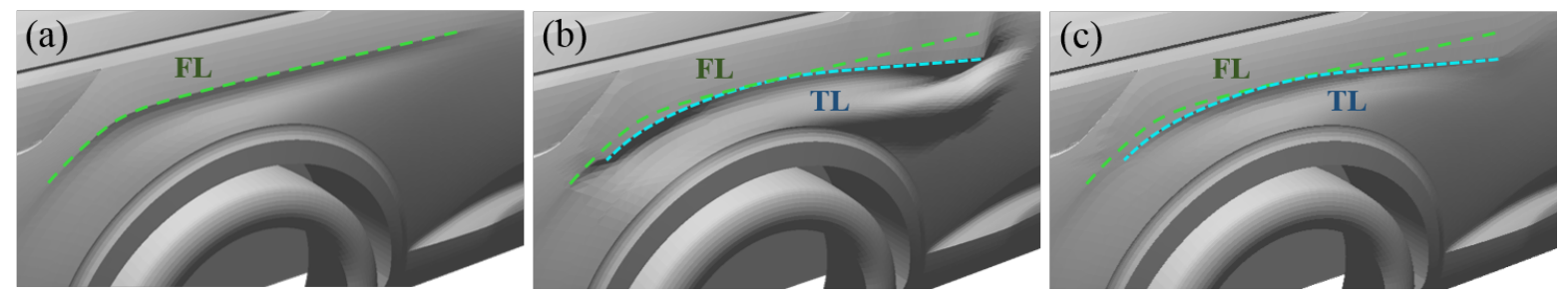

Figure 2: (a) Example of an on-surface FL modification applied to an industrial car model. The initial surface is deformed following the modification of FL (green) into TL (blue). (b) The use of surface deformation [8] highlights the bulging effect on the lower side of the TL, where the surface undergoes some compression. (c) This bulge disappears with our ASAN-FL method.

In contrast, polylines are rather used in stroke sketching systems $[27,20]$, appreciated by artists for loose and rough freehand drawings. As the quality of the line is a major point in our context, we choose to define the TL using a spline curve.

Surface deformation The following review focuses on whether the methods are suited or not for handling a smooth deformation guided by a feature line modification, knowing that the target line does not necessarily lie inside the initial surface.

The use of curve networks as a global control structure for guiding surface deformation is an appealing idea [28, 18, 45, 19]. However, curve networks are generally structured in order to define patch boundaries. In case where the feature line is not a patch boundary, which is often the case with FL as sharp lines surrounded by a smooth area, it is not obvious how to integrate it to the curve network. Also restricting the deformation to act locally is not naturally included.

Axial deformations $[45,51,29]$ are guided by an auxiliary curve. They therefore provide quite intuitive surface deformation tools. A shape deformation guided by a deforming feature line is conceptually similar and rather user-friendly as well. However, it calls for a specific deformation method, since the final surface is expected to interpolate the TL.

Combining sketch-based interfaces with subdivision surfaces, as proposed by Orbay and Kara [37], is another user-friendly shape design tool. The resulting surfaces are naturally smooth and of good visual quality. This type of representation is also used in industrial software such as Imagine and Shape by Dassault Systèmes [2]. Anyhow, surface modifications using subdivision surfaces require specific knowledge to monitor surface continuity across patches. Incorporation of feature lines into subdivision surfaces and interpolation of target lines are even more tedious to realize.

These difficulties also apply to Computer Aided Styling tools using B-spline based models where FL are often created as patch boundaries. In immersive software such as Gravity Sketch, the user defines the FL first and then builds patches according to these lines. Therefore, in order to modify the FL, one has to modify all the corresponding patches, which may largely extend a user-defined region of interest. While this parametric representation offers precise surface control, modifying a FL can quickly become a tedious process. Arbitrary deformation areas of interest and patch continuity conditions can be added as in Pernot et al. [39, 10], but it remains difficult to master smooth curvature distribution near the boundary of the deformation area of interest.

Mesh deformation and shape generation methods guided by sketched strokes within an immersive system, such as RodMesh [47] or Perkunder et al. [38], extending FiberMesh by Nealen et al. [36], and Teddy [23], create an inflated rotund shape, bounded by the closed user-defined 2D or 3D strokes. As the created surfaces all rely on optimization algorithms, the resulting shapes offer a smooth curvature distribution. Together with more intuitive interactions, these methods exhibit some appealing features also acceptable at the stage of design reviews. However, the round inflated shapes do not meet the requirements for FL modifications, such as open curves and local deformations mimicking manmade shapes.

Schemali et al. [42] proposed to use the so-called handle metaphor to deform the model and offered to select contour lines as handles. However, affine transfor- 


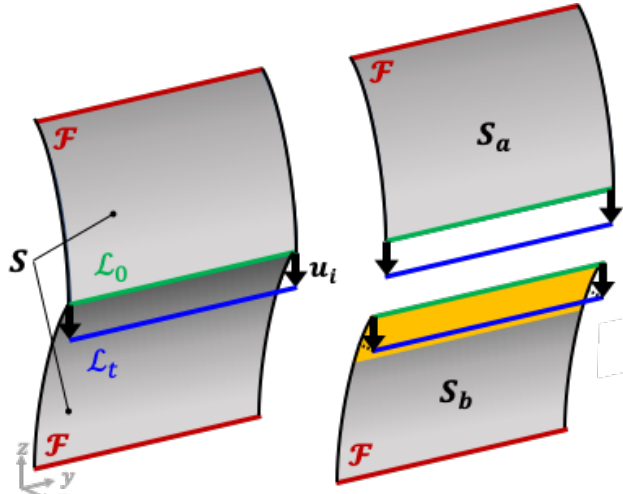

(a) (b)

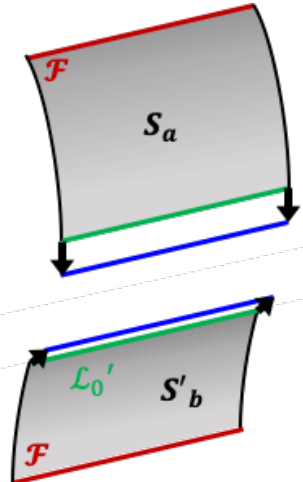

(c)

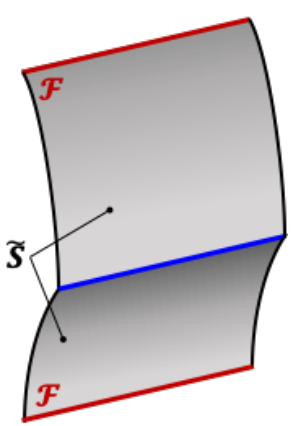

(d)

Figure 3: Feature line modification. (a) Subset of ROI with fixed constraints $(\mathcal{F})$, an initial FL, $\mathcal{L}_{0}$, and a TL, $\mathcal{L}_{t}$, of the category on-surface. (b) The sub-domains $\mathcal{S}_{a}$ and $\mathcal{S}_{b}$ on each side of $\mathcal{L}_{0}$ are processed independently and 'material' is removed (orange area) from $\mathcal{S}_{b}$, the sub-domain subjected to compression. This sub-domain is 'retracted'. (c) New FL $\mathcal{L}_{0}^{\prime}$ is defined by the boundary of the retracted sub-domain, $\mathcal{S}_{b}^{\prime}$, that is now submitted to tension. (d) Each sub-domain is deformed so that $\mathcal{L}_{0} \in \mathcal{S}_{a}$ and $\mathcal{L}_{0}^{\prime} \in \mathcal{S}_{b}^{\prime}$ coincide with $\mathcal{L}_{t}$.

mations only can be applied to these handles. Besides, they use the Linear Rotation Invariant (LRI) coordinates of Lipman et al. [32] to compute the deformation, which is better suited for organic shapes than for man-made ones. To keep the interaction intuitive while preserving the initial shape, Zhou et al. [54] proposed a two-step deformation. First, a deformation using a Laplacian or an As-Rigid-As-Possible (ARAP)-based method [46] is computed. Then, they compute a second minimization, with the energy of Eigensatz et al. [15, 14] which takes the discrete curvature into account. However, the use of discrete curvature might be sensitive to the mesh quality, which could be a downside when using industrial meshes.

To improve the surface deformations with curvature distribution adapted to man-made objects, physicallybased deformations $[46,8,9,30,39,31]$ provide the user with the handle metaphor, while enabling to monitor the stiffness distribution of the mechanical medium associated with the surface. Pernot et al. [39] proposed a membrane-like behavior restricted to tensile configurations, requiring additional stiffness monitoring and investigations regarding its behavior under large displacements. ARAP methods brought the description of finite rotations involved in membrane-like behaviors under large displacements. Stiffness distribution has emerged as an issue with approaches from [9, 30, 53, 31, 11]. Among them, the proposal of Le Vaou et al. [30] to monitor stiffness through the use of an anisotropic material with the ARAP-spokes and rims (ARAP-s\&r) method of Chao et al. [8] produces a satisfactory control of discrete curvature distribution.

However, the mechanical behavior of all these methods becomes inappropriate under specific loading configurations. Typically, membrane-like models, as discussed above, exhibit a buckling behavior under compression loading, producing bulging shapes, which is not desirable from an aesthetic standpoint. FL modification using these methods suffers from the same problem, in particular if the modification takes place "on" the surface (see Figure 2b). Consequently, more suited surface domain settings and loadings are required to process efficiently the FL modifications prescribed by designers.

In summary, most existing shape deformation methods guided by a curve (not always a FL), deal with off-surface TL. Methods able to deal with on-surface TL, such as Teddy [23] or FiberMesh [36] generate organic shapes, kind of inflated volumes, which are inappropriate for man-made designs. Physically-based methods, such as ARAP and its variants, are better suited for deformations guided by FL, as long as the deformation is off-surface, as observed through the upcoming sections. In case of on-surface TL, the resulting shapes are subject to unwanted buckling or wrinkling phenomena [5, 34]. As we will investigate in Section 3 , this effect can be explained mechanically by 
the occurrence of different loading conditions on either side of the FL, so that buckling or wrinkling naturally occurs in compressed areas.

We introduce in this paper a new method for shape deformation guided by a FL, which results in bulge-free shapes on both sides of the FL, whatever the type of FL deformations (off, on-surface). The idea is to combine the stiffness of the physically-based deformation process with a mesh retraction step in order to solve the challenging bulging problem.

\section{Method overview and initial settings}

\subsection{Feature line modification}

To achieve the modification of a FL in the context of (possibly immersive) design reviews, we identified the four following steps applied to a given surface mesh, which are illustrated in detail in Figure 3:

1 - user-selection of the initial FL, noted $\mathcal{L}_{0}$;

2 - user-selection of the deformation area around $\mathcal{L}_{0}$ that sets the fixed boundary, $\mathcal{F}:=\partial S$, and defines the region of interest $(\mathrm{ROI})$, noted $\mathcal{S}$, of the surface mesh;

3 - definition of the TL, noted $\mathcal{L}_{t}$;

4 - deformation of $\mathcal{S}$ to match $\mathcal{L}_{t}$. The resulting surface is noted $\widetilde{\mathcal{S}}$.

The main contribution of this paper addresses the last point: the deformation of $\mathcal{S}$. However, for the sake of completeness, Section 3.3 describes the processing of items 1 and 3 .

\subsection{Analysis of the bulging phenomenon and principle of the proposed ap- proach}

In order to avoid the bulging phenomenon depicted in Figure $2 b$, let us analyze possible mechanical models characterizing such undulations. The underlying structural model of ARAP-based and ASAN deformation methods is membrane-like model, i.e., a simplified model of non-linear membrane or shell, that can be subjected to loss of tension, an instability phenomenon that can produce wrinkles [34] or instabilities that can lead to the buckling phenomenon [5]. In either case, the characterization of these phenomena is not based on a single model $[5,34,33]$ and requires the access to a stress field to set inequalities as criterion. Monitoring wrinkles appears therefore as a topological optimization problem [34], which is out of range for an interactive operator, as presently required.

Another idea to prevent the bulging phenomenon could be to act over some parameters of the deformation model associated with $\mathcal{S}$ to modify the membrane stress field. Using ARAP-based and ASAN deformation methods, the available parameters would be the material, i.e., Young's modulus or Poisson's ratio, the boundary conditions or the domain shape of $\mathcal{S}$. Given the fact that boundary conditions are user-prescribed and cannot be modified (see Figure 3a), existing computer graphics approaches that prescribe Poisson's ratio $[8,30]$ and/or modify Young's modulus through anisotropy [30], do not enable to get rid of the bulging phenomenon.

The principle of the proposed approach lies instead in the modification of the domain shape of $\mathcal{S}$. This modification takes place through a decomposition of $\mathcal{S}$, i.e., a cut, into sub-domains $\left(\mathcal{S}_{a}\right.$ and $\mathcal{S}_{b}$, see Figure $3 b)$. This decomposition creates freedom to modify the shape of some sub-domain $\left(\mathcal{S}_{b}\right.$ in Figure $\left.3 \mathrm{c}\right)$ and corrects its stress state.

\subsection{Setting of $\mathcal{L}_{0}$ and $\mathcal{L}_{t}$}

Let us describe how users can define $\mathcal{L}_{0}$ and $\mathcal{L}_{t}$ into an immersive environment.

Selection of $\mathcal{L}_{0} \quad$ FL are considered as sharp lines only. In our context of car body design, the input model derives from a Computer Aided-Styling software, where sharp feature lines are located at the intersection (thus at the boundary) between some adjacent B-Spline patches. The tessellation of the surface patches that produces the surface mesh $\mathcal{S}$ results in edges that correspond exactly to the feature lines. Consequently, they appear in $\mathcal{S}$ as mesh edges with a sharp dihedral angle.

The mesh topology thus obtained from parametric models, and the fact that man-made shapes are generally precisely specified and free of noise, allow us to use the dihedral angle $\theta_{e}$ (see inset) to detect

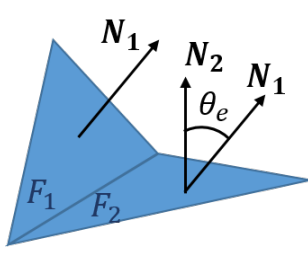
sharp lines. Applying the threshold procedure described in iWires [18], we get several sets of consecutive mesh 
edges, from which the user selects one as initial FL $\mathcal{L}_{0}$. Note that in case of other design contexts or noisy scanned input, FL detection can be addressed by a more robust method such as [22, 12, 49]. In all examples shown in the paper, the FL were automatically extracted from the models using a threshold angle of 10 degrees.

Definition of $\mathcal{L}_{t} \quad$ As a compromise between precision and intuition for the user, we propose to approximate $\mathcal{L}_{0}$ with a B-Spline curve and let the user modify it by moving its control points directly into the immersive environment, possibly combined with a curve fairing step [16] or using [35, 48, 13]. This simple setting is consistent with the approaches proposed to generate or modify curves into an immersive environment [21, 41, 4, 6]. User-friendly interaction with 3D geometry is still a challenging task, but not addressed in the scope of this contribution.

Once $\mathcal{L}_{t}$ is defined by the designer, we establish a correspondence between $\mathcal{L}_{0}$ and $\mathcal{L}_{t}$. This correspondence has to be smooth enough because large variations between the lines can produce undesirable physical phenomena, as discussed in Sections 4.2. The corresponding mapping projects each vertex $\mathbf{P}_{i} \in \mathcal{L}_{0}$ orthogonally onto $\mathcal{L}_{t}$. Usually, this mapping ends up shortening $\mathcal{L}_{0}$ and/or $\mathcal{L}_{t}$ at their ends (see Figure 4). This is however consistent with the fact that the extremities of $\mathcal{L}_{0}$ and $\mathcal{L}_{t}$ are regarded as sketched and should not be taken as hard constraints following [10]. Along the effective sequence of edges defining $\mathcal{L}_{0}$, we assume that the projection of every vertex $\mathbf{P}_{i}$ orthogonally to $\mathcal{L}_{t}$ is unique.

In the context of car body FL, this hypothesis is reasonable because modifications have a smooth curvature distribution and do not involve very large displacements from $\mathcal{L}_{0}$ to $\mathcal{L}_{t}$. Typically, for off-surface deformation, these deformations involve point displacements of $\mathcal{L}_{0}$ up to tens of millimeters of magnitude along the sur-

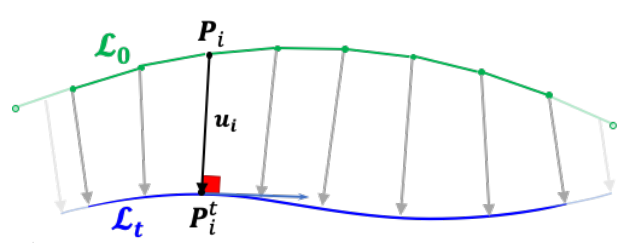

Figure 4: Displacement $\mathbf{u}_{i}$ : each vertex $\mathbf{P}_{i} \in \mathcal{L}_{0}$ is projected orthogonally onto $\mathcal{L}_{t}$ at $\mathbf{P}_{i}^{t}$. Solid lines indicate the effective definition interval of $\mathcal{L}_{0}$ and $\mathcal{L}_{t}$. Transparent edges of $\mathcal{L}_{0}$ and intervals of $\mathcal{L}_{t}$ are discarded when mapping $\mathcal{L}_{0}$ onto $\mathcal{L}_{t}$. face normal when $\mathcal{L}_{0}$ reaches several hundred millimeters length. Similarly, on-surface deformations involve displacements essentially located tangential to $\mathcal{S}$ with amplitudes close to $100 \mathrm{~mm}$ when $\mathcal{L}_{0}$ reaches nearly 400mm length.

As an alternative to the projection operator, an arclength parameterization of $\mathcal{L}_{t}$ could be used to set a correspondence between the vertices of $\mathcal{L}_{0}$ and $\mathcal{L}_{t}$. If this setting is more robust, it does not take well into account the shape changes between $\mathcal{L}_{0}$ and $\mathcal{L}_{t}$. Indeed, using an arc-length correspondence means that the discretization of $\mathcal{L}_{0}$ becomes independent of that of $\mathcal{L}_{t}$ and removes the link brought by the orthogonal projection at the risk of introducing mesh distortion that may create wrinkles. Throughout the rest of this paper, we use the projection operator to minimize the distortion of edges of $\mathcal{L}_{0}$ mapped onto $\mathcal{L}_{t}$.

Finally, this projection of $\mathbf{P}_{i} \in \mathcal{L}_{0}$ onto $\mathbf{P}_{i}^{t} \in \mathcal{L}_{t}$ defines the deformation constraints $\mathbf{u}_{i}$ when computing the final surface deformation:

$$
\mathbf{u}_{i}:=\mathbf{P}_{i}^{t}-\mathbf{P}_{i} .
$$

\section{4 "on/off-surface" FL deformation}

As stated already in Section 2 and shown in [44], variational mesh editing methods perform well in case of stretching or isometric deformations. However, in the case of FL modifications, shape compression will naturally occur and lead to undesired bulging effects (see an example in Figure 2b).

In this section, we will characterize this shape modification behavior by distinguishing two categories of FL modifications, one of which is the critical one. This distinction lets us understand and monitor the underlying mechanical phenomenon, which drives us directly to the solution we propose afterwards.

To fix some notations, let us assume that $\mathcal{L}_{0}$ enables the division of $\mathcal{S}$ into two adjacent surface sub-domains, denoted by $\mathcal{S}_{a}$ and $\mathcal{S}_{b}$, so that $\mathcal{S}=\mathcal{S}_{a} \cup \mathcal{S}_{b}$ (see Figure 5). $\mathcal{L}_{0}$ being inside $\mathcal{S}$, we artificially extend $\mathcal{L}_{0}$ up to the border $\mathcal{F}$ of $\mathcal{S}$. The extension of $\mathcal{L}_{0}$ is denoted $\widehat{\mathcal{L}}_{0}$ and $\mathcal{L}_{0} \subset \widehat{\mathcal{L}}_{0}, \mathcal{S}_{a} \cap \mathcal{S}_{b}=\widehat{\mathcal{L}}_{0}$ (more details in Sections 4 and 5).

Let us now distinguish two categories of FL modifications according to whether the displacements $\mathbf{u}_{i}$ are nearly orthogonal or parallel to the surface $\mathcal{S}$. The relative positions of $\mathcal{L}_{t}$ and $\mathcal{L}_{0}$ with respect to $\mathcal{S}$ enable a qualitative mechanical distinction as follows: 


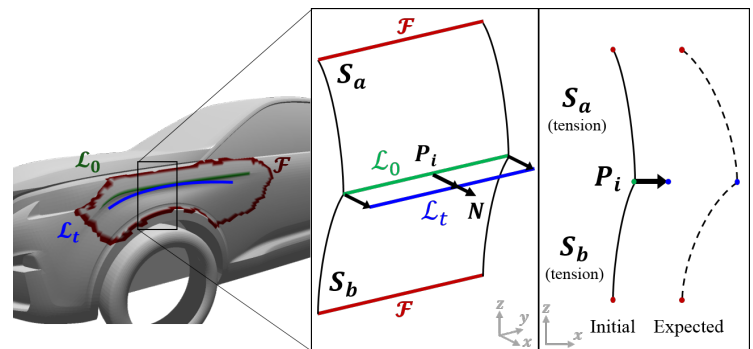

(a) $\mathcal{L}_{t}$ outside the surface

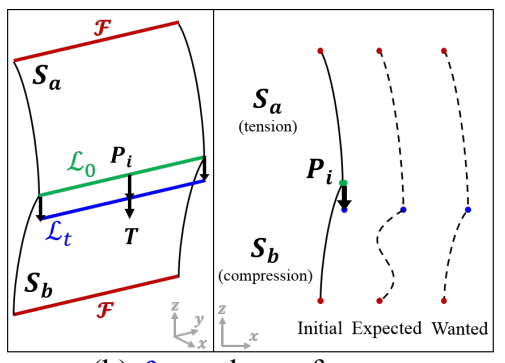

(b) $\mathcal{L}_{t}$ on the surface

Figure 5: Two categories of FL modification $\mathcal{L}_{0}$ into $\mathcal{L}_{t}$. (a) off-surface deformation where displacements $\mathbf{u}_{i}$ of $\mathcal{L}_{0}$ follow approximately the average normal $\mathbf{N}$ : sections of $\mathcal{S}_{a}$ and $\mathcal{S}_{b}$ get elongated, (b) on-surface deformation, where displacements $\mathbf{u}_{i}$ of $\mathcal{L}_{0}$ follow approximately the average tangent $\mathbf{T}$ : sections of $\mathcal{S}_{a}$ get elongated, those of $\mathcal{S}_{b}$ 's undulate upon compression whereas the designer wants to produce tensile curves.

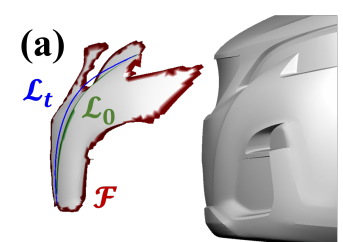

ROI

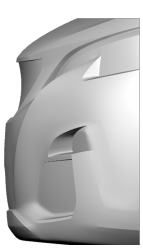

ASAN

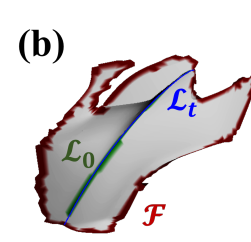

ROI

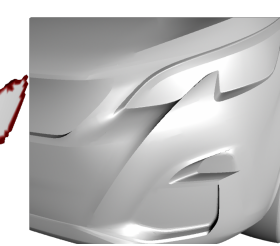

Initial

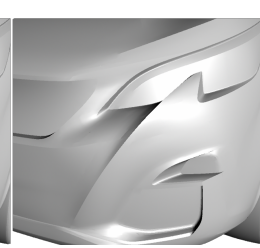

ASAN

Figure 6: An example of off-surface deformation. (a) Side view and (b) front view. Since the surface undergoes mostly tension loads, ASAN method gives a satisfactory result.
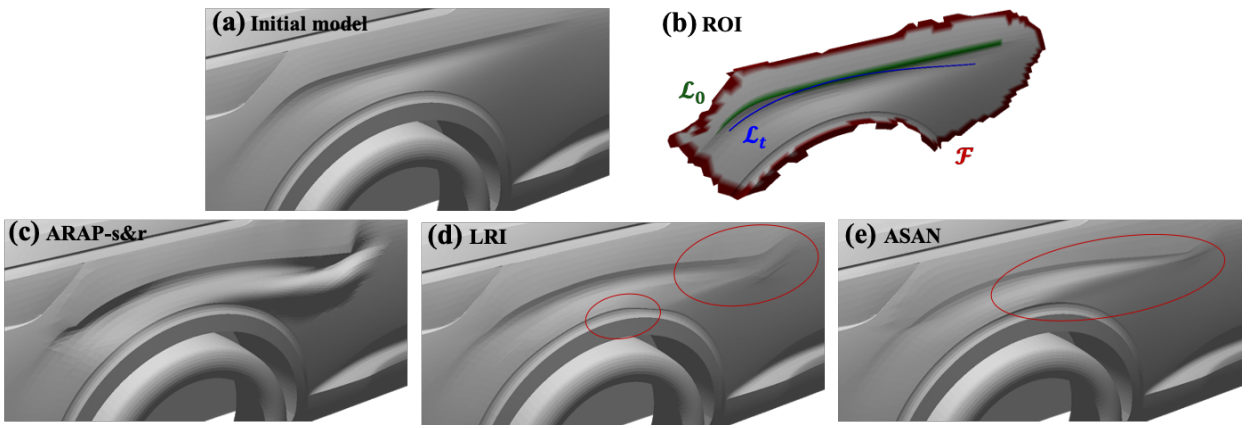

Figure 7: on-surface TL achieved with surface deformations producing a bulging effect because of the existence of a compression loading in the area located under $\mathcal{L}_{t}$. (a) Initial model with $\mathcal{L}_{0}$. (b) User-defined $\mathcal{S}$ with $\mathcal{L}_{0}$ and $\mathcal{L}_{t}$. (c) Deformed surface $\widetilde{\mathcal{S}}$ obtained with the isotropic membrane-like ARAP s\&r method exhibiting a bulging effect under $\mathcal{L}_{t}$. (d) $\widetilde{\mathcal{S}}$ obtained with LRI showing non smooth curvature varying areas (circled in red). (e) $\widetilde{\mathcal{S}}$ obtained with anisotropic membrane-like deformation ASAN still exhibiting a non smooth curvature varying area (circled in red) under $\mathcal{L}_{t}$. 
- "off-surface": $\mathcal{L}_{t}$ is said off-surface if there exists a direction $\mathbf{N}$ approximating the normal of $\mathcal{S}$ at $\mathbf{P}_{i}$, such that $\forall i, \mathbf{u}_{i} \approx \mathbf{N}$. This configuration is illustrated in Figure 5a. There, $\mathcal{S}_{a}$ and $\mathcal{S}_{b}$ are subjected to similar global stress states: both are under a tension configuration. In this case, a deformation method offering an appropriate stiffness distribution, such as ASAN [30] or ARAP- $n^{1}$ [9], produces an appropriate result (see Figure 6);

- "on-surface": $\mathcal{L}_{t}$ is on-surface if there exists a direction $\mathbf{T}$ approximating a tangent to $\mathcal{S}$ at $\mathbf{P}_{i}$, such that $\forall i, \mathbf{u}_{i} \approx \mathbf{T}$ (see Figure 5b). Normal sections of $\mathcal{S}_{a}$ and $\mathcal{S}_{b}$ along $\mathcal{L}_{0}$ are subjected to different stress states: sections of $\mathcal{S}_{a}$ are elongated while those of $\mathcal{S}_{b}$ shrink, letting $\mathcal{S}_{b}$ subjected to compression from a mechanical standpoint. In a general setting, $\mathcal{S}_{a}$ and $\mathcal{S}_{b}$ can be swapped but one of them will always be subjected to compression. A mechanically sound deformation method will lead to an expected but undesirable bump because of the compression loading applied to either side of $\mathcal{L}_{t}$.

In summary, if the target line $L_{t}$ is off-surface, the boundary conditions are pulling both sub-domains, whereas if the target line $L_{t}$ is on-surface, compression is likely to occur on one side of the surface.

In the configuration of on-surface modification of $\mathcal{L}_{0}$, increasing the stiffness of the membrane-like ${ }^{2}$ mechanical model associated with $\mathcal{S}$ could be thought as an alternative, but turns out to not give satisfying results. To show this, we implemented state-of-the-art membranelike deformation methods: the basic ARAP, and variants which tend to increase stiffness, such as ARAP s\&r [8], ARAP- $n$ [9] and ASAN [30], as well as LRI [32]. The bump reduces but does not disappear (see Figure 7). ARAP- $n$ [9] exhibits some bulging effect near $\mathcal{L}_{t}$ that cannot be removed, even with a large vertex neighborhood, i.e., $n>20$, (see Section 6). Turning the membrane-like model into an effective shell-like $\mathrm{e}^{3}$ could be part of a solution but at the increase of complexity and processing time. As a result, on-surface deformation of $\mathcal{L}_{0}$ requires specific processing.

The proposed method preserves the membrane-like model but modifies the compression loading so that it

\footnotetext{
${ }^{1}$ The extension of ARAP-spokes\&rims [8] proposed by Chen et al. noted here ARAP- $n$.

${ }^{2}$ Membrane-like mechanical models include all the variational surface deformation models [46, 8, 9, 30, 31, 52].

${ }^{3}$ Shell-like mechanical models require Finite Element simulations with volume elements.
}

turns into a traction loading. Because the mechanical stress states on each side of $\mathcal{L}_{t}$ are different, we process $\mathcal{S}_{a}$ and $\mathcal{S}_{b}$ separately. Let us denote in the rest of the paper by $S_{a}$ the side subjected to tension loading, which does not require specific processing. We apply a pre-processing step, a mesh retraction, to the surface part $\mathcal{S}_{b}$ under compression, so that the on-surface FL modification finally acts as a traction loading on both sides.

\subsection{Algorithm overview (ASAN-FL)}

Our goal is to propose a mesh deformation method that is able to deform a feature line $\mathcal{L}_{0}$ while performing well for man-made free-form shapes. More particularly, the deformation method should preserve the visual aspect of the feature line, i.e. the initial dihedral angle along $\mathcal{L}_{0}$. For off-surface configurations, ASAN [30] is able to do so. For on-surface configurations, we propose a custom treatment to solve the bulging effect, before deforming the mesh to meet the target line $\mathcal{L}_{t}$.

Our algorithm, called ASAN for Feature Lines $(A S A N-F L)$ composes of the following steps (see Figure 8):

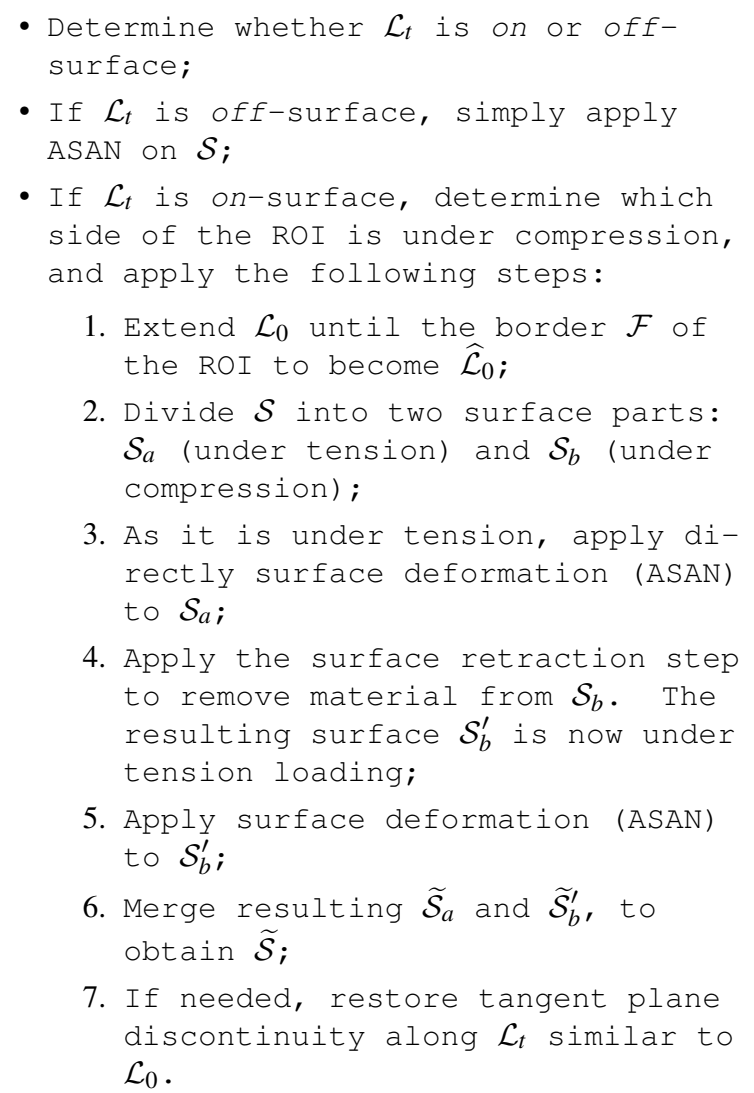




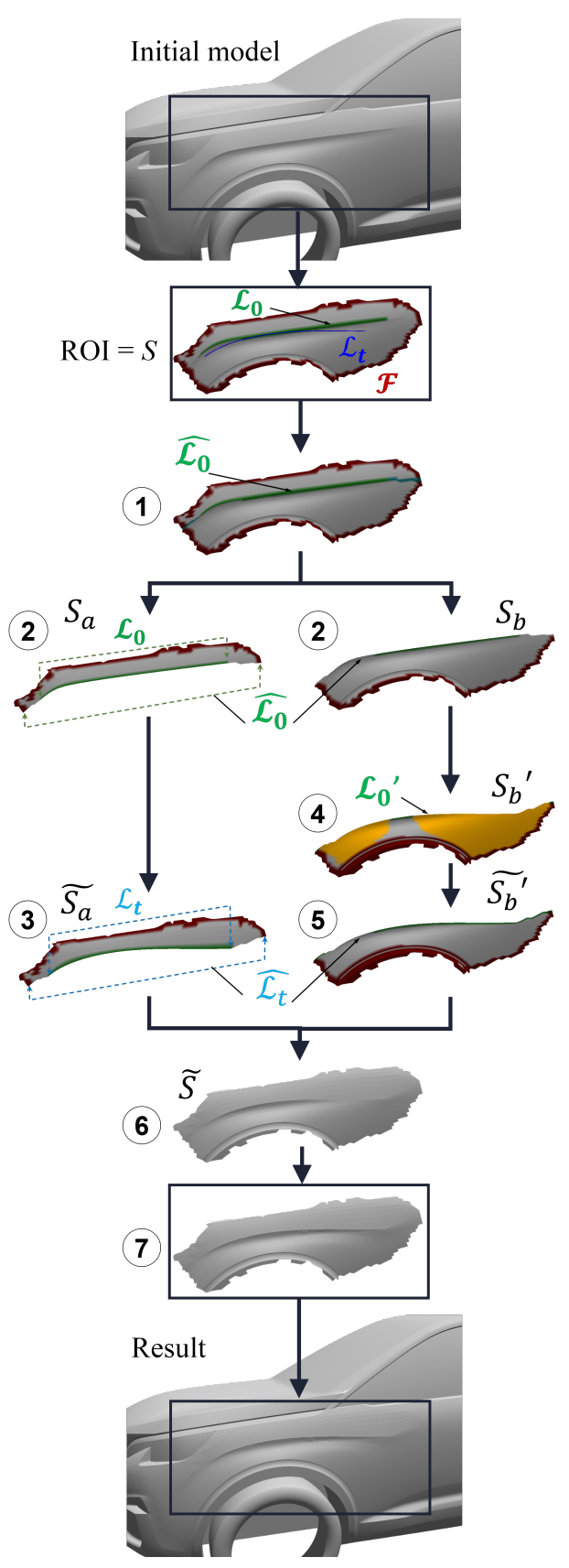

Figure 8: The steps of ASAN-FL. When $\mathcal{L}_{t}$ is on-surface, $\mathcal{S}$ is divided into two regions $\mathcal{S}_{a}$ and $\mathcal{S}_{b}$. (1) $\mathcal{L}_{0}$ is extended to (2) distinguish $\mathcal{S}_{a}$ (under tension) from $\mathcal{S}_{b}$ (under compression). (3) ASAN deformation is applied to $\mathcal{S}_{a}$. (4) To allow the surface to be subjected to traction loading, the retraction step is applied to $\mathcal{S}_{b}$ before applying ASAN deformation (5). (7) a complementary ASAN deformation can be applied to restore the normal discontinuity along $\mathcal{L}_{0}$.
The next section describes our mesh retraction method of step 4 as the key ingredient of our algorithm. The other steps of our algorithm are then presented in Section 5.

\section{Mesh retraction}

Let us consider the reference case where $\mathcal{S}_{b}$ is subject to compression loading (see Section 3.4), we propose to modify $\mathcal{S}_{b}$ using a "material" removal principle that produces $\mathcal{S}_{b}^{\prime}$, so that when $\mathcal{S}_{b}^{\prime}$ deforms to meet $\mathcal{L}_{t}, \mathcal{S}_{b}^{\prime}$ is subjected to a traction loading. Figure 3 summarizes this process.

Such virtual material removal could be obtained in different ways. To remove material from $\mathcal{S}_{b}$ and obtain a surface $\mathcal{S}_{b}^{\prime}$ under tension loading, one straightforward possibility would be to cut-off some mesh elements, where $\mathcal{L}_{t}$ projects onto $\mathcal{S}_{b}$. However, a projection may not be robust on free-form surfaces. Moreover, the cutting operator modifies the sequence of edges at the boundary of $\mathcal{S}_{b}$, requiring a remeshing process that can introduce side-effects during the deformation process. We therefore implement a mesh sliding method, which preserves the initial mesh topology while removing material (by reducing the surface area) to produce $\mathcal{S}_{b}^{\prime}$.

Criteria for an appropriate transformation of $\mathcal{S}_{b}$ The desired process is set up using the following preservation criteria:

$\left(c_{1}\right)$ Shape preservation: to avoid introducing unwanted shape irregularities during mesh transformation;

$\left(c_{2}\right)$ Topology preservation: to ease the merge of $\widetilde{\mathcal{S}}_{a}$ and $\widetilde{\mathcal{S}}_{b}^{\prime}$ along $\mathcal{L}_{t}$ after the deformation process of $\mathcal{S}_{a}$ and $\mathcal{S}_{b}^{\prime}$;

$\left(c_{3}\right)$ Symmetry preservation: to preserve, if applicable, mesh ROI symmetry properties.

Sliding method As a starting point, we chose the sliding method proposed by Pinskiy [40], which enables mesh vertices to slide over the polygonal surface while keeping its initial visual aspect. This approach is well-suited as it is shape and topology preserving (criteria $c_{1}, c_{2}$ ) without requiring global parameterization and remeshing. However, Pinskiy's method only works pointwise, whereas we need to process the whole FL to 


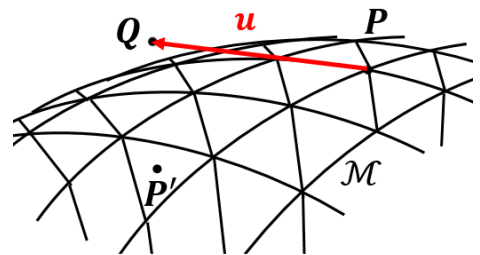

(a)

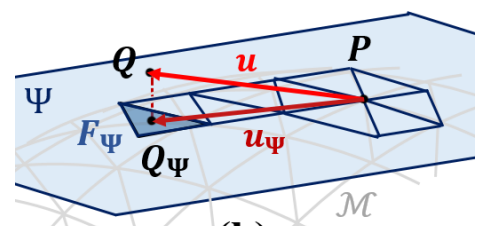

(b)

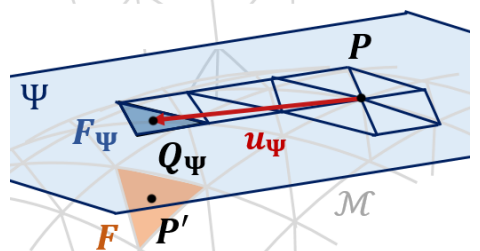

(c)

Figure 9: (a) The sliding moves $\mathbf{P}$ towards a point $\mathbf{Q}$ while staying on $\mathcal{M}$. (b) To obtain the slid position $\mathbf{P}^{\prime}$, use $\Psi$, the local tangent space of $\mathbf{P}$ where $\mathbf{Q}$ is projected into $\mathbf{Q}_{\Psi}$. (c) $\mathbf{P}^{\prime}$ is located into $F$ using the barycentric coordinates of $\mathbf{Q}_{\Psi}$ wrt. $F_{\Psi}$.
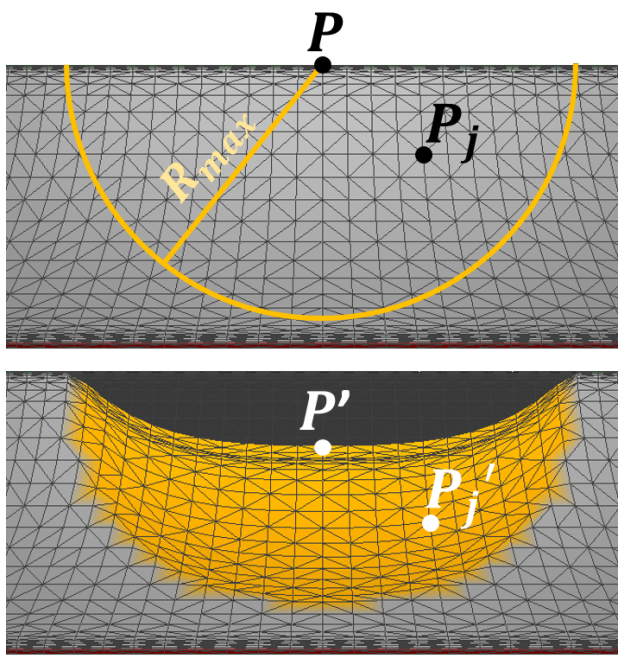

Figure 10: (Top) Pinskiy [40] defines an active area around $\mathbf{P}$ that gathers every vertex $\mathbf{P}_{j}$ within a radius $R_{\max }$ of $\mathbf{P}$. (Bottom) Point $\mathbf{P}$ and points $\mathbf{P}_{j}$ in the active area slide to their new position (resp. $\mathbf{P}^{\prime}$ and $\mathbf{P}_{j}^{\prime}$ ). be slid. In the following, we explain why using this sliding method sequentially for all mesh vertices on $\mathcal{L}_{0}$ is not a good alternative in our context. We rather propose an extension of Pinkiy's method, where all vertices are slid simultaneously in order to meet all criteria.

\subsection{Sliding of a point $P$ towards a point $P^{\prime}$}

For convenience, let us briefly recall here the main steps of Pinskiy's method. In this explanation, let us note $\mathcal{M}$ the surface mesh. The purpose of Pinskiy's method is to slide a surface point $\mathbf{P} \in \mathcal{M}$ over the surface $\mathcal{M}$ under a prescribed 3D displacement $\mathbf{u}$ of $\mathbf{P}, \mathbf{Q}:=\mathbf{P}+\mathbf{u}$ (see Figure 9(a)). The goal is not only to compute the destination point $\mathbf{P}^{\prime} \in \mathcal{M}$ but also to compute a consistent and intuitive sliding for all vertices in a certain neighborhood, in order to keep the mesh free of overfoldings. Pinskiy proposed the following steps:

- define a tangent plane $\Psi$ at $\mathbf{P}$;

- project the point $\mathbf{Q}$ orthogonaly onto $\Psi$, which defines $\mathbf{Q}_{\Psi}$ and a displacement $\mathbf{u}_{\Psi}$ (see Fig. 9(b));

- starting from $\mathbf{P}$, the neighboring faces are locally parameterized in $\Psi$, until the face $F_{\Psi}$ containing $\mathbf{Q}_{\Psi}$ is found (see Fig. 9(c));

- compute the barycentric coordinates of $\mathbf{Q}_{\Psi}$ with respect to $F_{\Psi}$

- use these coordinates from $F_{\Psi}$ with respect to the corresponding face $F$ of $\mathcal{M}$ to compute $\mathbf{P}^{\prime} \in \mathcal{M}$.

Active area around $\mathbf{P}$ Pinskiy then defines an active area around this principal vertex $\mathbf{P}$ within a region radius $R_{\max }$ in $3 \mathrm{D}$, in order to determine its neighboring sliding vertices $\mathbf{P}_{j}$ (see Figure 10). The displacement magnitudes of $\mathbf{P}_{j}$ decrease with their distance to $\mathbf{P}$ using a non-linear decreasing damping function. This active area ensures that vertices $\mathbf{P}_{j}$ around the principal vertex $\mathbf{P}$ are slid consistently. A complete description can be found in [40].

\subsection{Sequential sliding}

Sliding of a whole polyline In our setting, we need to slide not only a single vertex $\mathbf{P}$ but all the vertices $\mathbf{P}_{i}$ of the polyline $\widehat{\mathcal{L}_{0}}$. When applying ASAN to $\mathcal{S}_{a}$ (see step 3 in Fig. 8), we deform $\mathcal{L}_{0}$ to match $\mathcal{L}_{t}$. The deformed version of $\widehat{\mathcal{L}_{0}}$ on $\mathcal{S}_{a}$ is the extension of $\mathcal{L}_{t}$ and noted $\widehat{\mathcal{L}_{t}} . \widehat{\mathcal{L}}_{t}$ is the target of the sliding process applied to $\widehat{\mathcal{L}_{0}}$. 


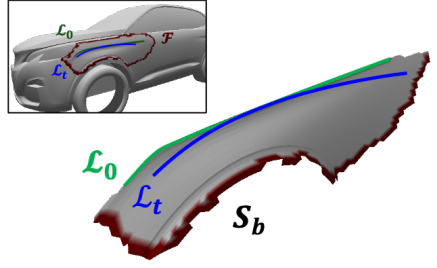

(a) Initial mesh

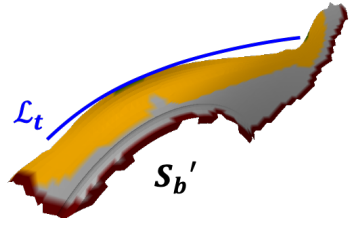

(b) Sequential sliding

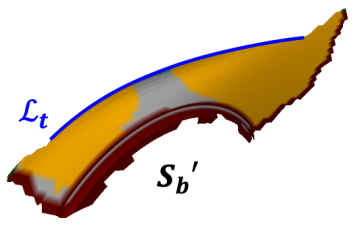

(c) Retraction

Figure 11: Effects of the sequential sliding and the retraction on an industrial example. (a) Initial setting with $\mathcal{L}_{0}, \mathcal{L}_{t}$ and the sub-domain $\mathcal{S}_{b} \subset \mathcal{S}$. (b) $\mathcal{S}_{b}^{\prime}$ obtained with the sequential sliding. The orange area indicates the area covered by the successive areas of influence where vertices locations get modified. (c) $\mathcal{S}_{b}^{\prime}$ produced by the retraction process. The orange area indicates the mesh area where mesh vertices are slid.

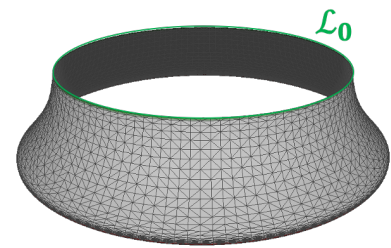

(a) Initial mesh

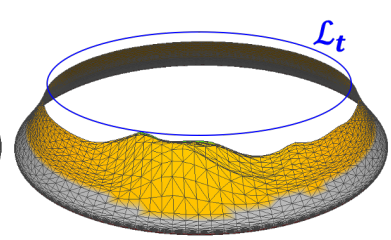

(b) Sequential sliding

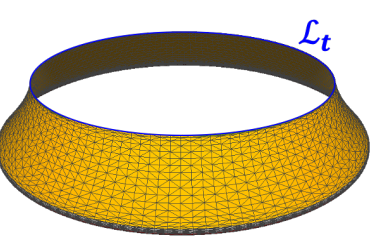

(c) Retraction

Figure 12: Effect of the sequential sliding and the retraction on an academic example extracted from a chess part. (a) Initial setting with $\mathcal{L}_{0}$ and the initial sub-domain that is close to axi-symmetry. (b) The user-prescribed position of $\mathcal{L}_{t}$ and the resulting domain obtained with the sequential sliding. The orange area indicates the area covered by the successive areas of influence where vertices locations get modified. The resulting domain loses the near axi-symmetric property of the initial domain. (c) Result produced by the retraction process. The orange area indicates the mesh area where mesh vertices are slid. The retracted domain is still close to axi-symmetry.

We thus need to slide the vertices $\mathbf{P}_{i}$ of $\widehat{\mathcal{L}_{0}}$ towards their corresponding vertices $\mathbf{P}_{i}^{t} \in \widehat{\mathcal{L}_{t}}$.

This prescription is justified by the fact that the displacements of vertices along $\widehat{\mathcal{L}_{0}}$ in $\mathcal{S}_{a}$ are subjected to its mechanical deformation, a physical phenomenon of same type as the one that will be applied later to the slid surface $\mathcal{S}_{b}^{\prime}$. This is ensuring a high quality continuity across the common boundary of $\widetilde{\mathcal{S}}_{a}$ and $\widetilde{\mathcal{S}}_{b}^{\prime}$.

The prescribed displacements are therefore given by $\mathbf{u}_{i}=\mathbf{P}_{i}^{t}-\mathbf{P}_{i}^{\prime}$. The slid points are noted $\mathbf{P}_{i}^{\prime} \in \mathcal{S}_{b}$ and belong to $\mathcal{L}_{0}^{\prime}$, the slid location of $\widehat{\mathcal{L}}_{0}$, which forms the boundary of $\mathcal{S}_{b}^{\prime}$ (see Fig. 8).

Sequential sliding A first possibility would be to apply the displacement of each vertex $\mathbf{P}_{i} \in \widehat{\mathcal{L}}_{0}$ sequentially along $\widehat{\mathcal{L}}_{0}$. However, the active area around $\mathbf{P}_{i}$ incorporates vertices $\mathbf{P}_{k}$ that belong to $\widehat{\mathcal{L}}_{0}$ as well. When $\mathbf{P}_{k}$ becomes the principal vertex, its displacement $\mathbf{u}_{k}$ has to be updated into $\mathbf{u}_{k}^{\text {new }}$ to take into account the new position $\mathbf{P}_{k}^{\text {new }}$. The sliding is then computed with the new prescribed displacement $\mathbf{u}_{k}^{\text {new }}$ :

$$
\mathbf{u}_{k}^{\text {new }}=\mathbf{P}_{k}^{t}-\mathbf{P}_{k}^{\text {new }} \text {. }
$$

After processing all the points of $\widehat{\mathcal{L}}_{0}$ under a given $R_{\text {max }}$, a surface $\mathcal{S}_{b}^{\prime}$ is produced that lies inside $\mathcal{S}_{b}$. $\mathcal{S}_{b}^{\prime}$ is a slid version of $\mathcal{S}_{b}$ and is subjected to a tension stress state when applying the ASAN deformation.

Limitations of the sequential sliding This sequential approach faces several limitations. Firstly, the use of an active area means that one must define its radius $R_{\text {max }}$. In [40], Pinskiy considers the specific context of character animation: he thus lets the rigger, who prepares the model for the animator, define the appropriate active area radii. However, our context requires an automatic definition of the extent of the sliding on $\mathcal{S}_{b}$ to preserve the intuitiveness of the FL adjustment. In particular, the sliding process must take into account $\mathcal{F}$, the ROI boundary (possibly close to $\mathcal{L}_{t}$ ) to avoid moving vertices outside the ROI. 


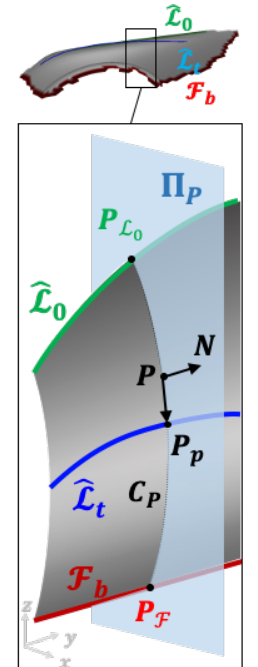

Figure 13: The projection $\mathbf{P}_{p}$ of $\mathbf{P}$ on $\mathcal{L}_{t}$ defines a direction $\mathbf{v}_{\text {proj }}$, combined with the normal $\mathbf{N}$ at $\mathbf{P}$ to define the plane $\Pi_{P}$. Then, the intersection of $\Pi_{P}$ with the boundary of $\mathcal{S}_{b}$ defines the point $\mathbf{P}_{\mathcal{L}_{0}}$ along $\widehat{\mathcal{L}}_{0}$ and $\mathbf{P}_{\mathcal{F}}$ along $\mathcal{F}_{b}$.

Moreover, this sequential sliding is obviously orderdependent, and the displacements of $\mathbf{P}_{k} \in \widehat{\mathcal{L}}_{0}$ add up throughout this sliding process, possibly producing unwanted accumulations of vertices and hence, mesh distortions. We applied this sequential sliding to two examples, a real world example and an academic one, where artefacts, such as unwanted accumulations in Figure 11(b) and loss of ROI symmetry in 12(b) can be observed.

From a mechanical standpoint, the sequential sliding may face difficulties regarding the underlying stressstrain fields of some ARAP or ASAN-based deformation. Indeed, Figure 11 shows that the slid configuration of $\widehat{\mathcal{L}_{0}}$ may deviate from $\mathcal{L}_{t}$ significantly enough to create unbalanced stress states, creating wiggles on $\mathcal{S}_{b}^{\prime}$ as depicted in Figure 20(g). As a result, it is important that the modification process of $\mathcal{S}_{b}$ produces a boundary of $\mathcal{S}_{b}^{\prime}$ that stays as close as possible to $\mathcal{L}_{t}$.

\subsection{Retraction of $\mathcal{S}_{b}$ into $\mathcal{S}_{b}^{\prime}$}

Rather than sliding sequentially each vertex $\mathbf{P}_{i} \in \widehat{\mathcal{L}}_{0}$ and its active area, which creates dependencies through overlapping active areas, a better solution would be to compute the displacement of all vertices of $\mathcal{S}_{b}$ simultaneously and homogeneously, according to their distance to the boundary of $\mathcal{S}_{b}$, noted $\partial \mathcal{S}_{b}$. This sliding process is called retraction and uses $\widehat{\mathcal{L}}_{t}$ as set in Section 4.2.

$\partial \mathcal{S}_{b}$ is decomposed into two polylines $\mathcal{F}_{b}$ and the previously defined $\widehat{\mathcal{L}_{0}}$ such that (see Figure 13 ):

$$
\mathcal{F}_{b}=\mathcal{S}_{b} \cap \mathcal{F} \quad \text { and } \quad \partial \mathcal{S}_{b}=\mathcal{F}_{b} \cup \widehat{\mathcal{L}_{0}} .
$$

Then, considering an arbitrary point $\mathbf{P}$ of $\mathcal{S}_{b}$, the idea is to slide $\mathbf{P}$ with a large displacement if $\mathbf{P}$ is close to $\widehat{\mathcal{L}}_{0}$ and a small one when $\mathbf{P}$ gets close to $\mathcal{F}_{b}$. Therefore, we propose to weight the displacement of each vertex $\mathbf{P}$ in accordance with the distances of $\mathbf{P}$ to $\widehat{\mathcal{L}_{0}}$ and $\mathcal{F}_{b}$, respectively. Since these displacements will shrink $\mathcal{S}_{b}$, we call this method the retraction of $\mathcal{S}_{b}$ into $\mathcal{S}_{b}^{\prime}$.

Computing the displacements $\mathbf{u}_{P} \quad$ Firstly, let us consider that the curve $\mathcal{L}_{t}$ is extended at its extremities with its tangents. $\mathbf{P} \in \mathcal{S}_{b}$ is projected orthogonaly onto $\mathcal{L}_{t}$ as $\mathbf{P}_{p}$ (see Figure 13). Then, $\mathbf{P}_{p}$ and $\mathbf{P}$ set the projection direction $\mathbf{v}_{\text {proj }}$ as:

$$
\mathbf{v}_{p r o j}=\frac{\mathbf{P}_{p}-\mathbf{P}}{\left\|\mathbf{P}_{p}-\mathbf{P}\right\|},
$$

The plane $\Pi_{P}$ is defined by $\left(\mathbf{v}_{\text {proj }}, \mathbf{N}\right)$ where $\mathbf{N}$ is the unit normal of $\mathcal{S}_{b}$ at $\mathbf{P}$. Extending $\mathcal{L}_{t}$ with its tangents is a means to ensure the existence of $\mathbf{P}_{p}$ for every $\mathbf{P} . \mathcal{S}_{b}$ is subjected to the following hypothesis:

(H) The set of curves $\mathbf{C}_{P}=\Pi_{P} \cap \mathcal{S}_{b}, \forall P \in \mathcal{S}_{b}$, has no intersection.

This hypothesis is a major one to prevent displacements $\mathbf{u}_{P}$ to generate overfoldings of the retracted surface $\mathcal{S}_{b}^{\prime}$. Commonly, $(\mathrm{H})$ holds because $\mathcal{S}_{b}$ is smoothly varying and is close to a cylindrical or planar area. Further, $\mathcal{L}_{t}$ is close to $\mathcal{S}_{b}$ ("on"-surface TL) and smoothly varying too. Then, $\Pi_{P}$ is the normal plane of $\mathcal{L}_{t}$ at $\mathbf{P}_{p}$. This is heading toward a curvature centre of $\mathcal{L}_{t}$ far from $\mathcal{S}_{b}$, which is a property enforcing the non intersection of the curves $\mathbf{C}_{P}$

Secondly, let us define the following points, $\mathbf{P}_{\mathcal{L}_{0}}$ (resp. $\mathbf{P}_{\mathcal{F}}$ ) as the intersection points between $\Pi_{P}$ and $\widehat{\mathcal{L}}_{0}\left(\right.$ resp. $\mathcal{F}_{b}$ ), as shown in Figure 13:

$$
\mathbf{P}_{\mathcal{L}_{0}}=\Pi_{P} \cap \widehat{\mathcal{L}}_{0} \quad \text { and } \quad \mathbf{P}_{\mathcal{F}}=\Pi_{P} \cap \mathcal{F}_{b} .
$$

The existence of $\mathbf{P}_{\mathcal{L}_{0}}$ and $\mathbf{P}_{\mathcal{F}}$ is enforced by construction of $\mathcal{S}_{b}$, bounded by $\widehat{\mathcal{L}}_{0}$ and $\mathcal{F}_{b}$, and by $(\mathrm{H})$. However, there may be areas of $\mathcal{S}_{b}$ where either $\mathbf{P}_{\mathcal{L}_{0}}$ or $\mathbf{P}_{\mathcal{F}}$ does not exist. In this case, $\mathbf{u}_{P}=\mathbf{0}$.

Regarding the uniqueness of $\mathbf{P}_{\mathcal{L}_{0}}$ and $\mathbf{P}_{\mathcal{F}}$, the contours of the ROI usually originate from a smooth movement of the designer around $\mathcal{L}_{t}$ and when $\Pi_{P}$ moves 


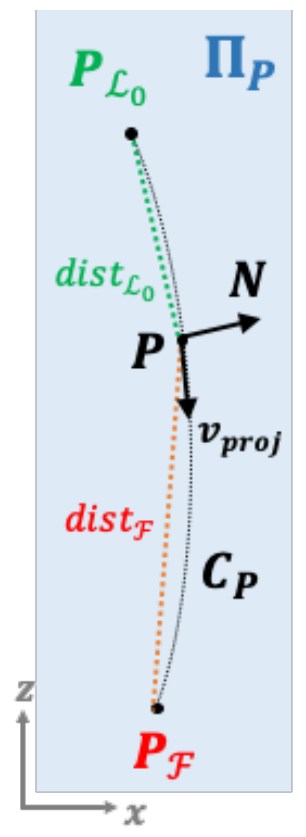

Figure 14: Euclidean distances between $\mathbf{P}$ and $\mathbf{P}_{\mathcal{L}_{0}}$ and $\mathbf{P}$, respectively, define the displacement $\mathbf{u}_{P}$ of $\mathbf{P}$.

along the vertices of $\widehat{\mathcal{L}}_{0}, \mathbf{P}_{\mathcal{L}_{0}}$ and $\mathbf{P}_{\mathcal{F}}$ are found unique (see Figure 8). However, $\mathcal{S}_{b}$ can get more complex (see Figure 16(b)) and lead to multiple solutions for $\mathbf{P}_{\mathcal{F}}$ in particular. In this setting, the solution closest to $\mathbf{P}$ is chosen. Such complex configurations of $\mathcal{S}_{b}$ express discontinuities of the distances to $\mathbf{P}$. Anyhow, as long as these discontinuities are far enough from $\widehat{\mathcal{L}}_{0}$, they have no significant impact on $\mathcal{S}_{b}^{\prime}$.

Finally, the following Euclidean distances (shown in Fig. 14) are defined:

$$
\operatorname{dist}_{\mathcal{L}_{0}}=\left\|\mathbf{P}_{\mathcal{L}_{0}}-\mathbf{P}\right\| \quad \text { and } \quad \operatorname{dist}_{\mathcal{F}}=\left\|\mathbf{P}_{\mathcal{F}}-\mathbf{P}\right\|
$$

to set the weight $\lambda$ :

$$
\lambda=\frac{\operatorname{dist}_{\mathcal{F}}}{\operatorname{dist}_{\mathcal{L}_{0}}+\operatorname{dist}_{\mathcal{F}}},
$$

taking values in the interval $[0,1]$. As a result, we define the displacement $\mathbf{u}_{P}$ such that:

$$
\mathbf{u}_{P}=\lambda \mathbf{u}_{0},
$$

where $\mathbf{u}_{0}$ is the displacement prescribed at $\mathbf{P}_{\mathcal{L}_{0}}$. The closer $\mathbf{P}$ is to $\widehat{\mathcal{L}}_{0}$, the closer $\lambda$ is to one, which ensures that $\mathbf{P}$ gets as close as possible to $\mathcal{L}_{t}$. To define $\mathbf{u}_{0}$, we use the displacements of $\mathbf{P}_{i}$ and $\mathbf{P}_{i+1}$, the extremities of the edge of $\widehat{\mathcal{L}}_{0}$ where $\mathbf{P}_{\mathcal{L}_{0}}$ is located. We take the barycentric coordinate $\alpha \in[0,1]$ of $\mathbf{P}_{\mathcal{L}_{0}}$ on $\left[\mathbf{P}_{i}, \mathbf{P}_{i+1}\right]$ :

$$
\mathbf{u}_{0}=\alpha \mathbf{u}_{i}+(1-\alpha) \mathbf{u}_{i+1} \text {. }
$$

If the intersection curve between $\mathcal{S}_{b}$ and $\Pi_{P}$ reduces to a line segment, the weight $\lambda$ reduces to a linearly decreasing function wrt to the location of $\mathbf{P}$ along that segment. Further, under these circumstances, it can be shown that the sliding of any point $\mathbf{P}$ is a monotonous decreasing function of its distance wrt to $\mathbf{P}_{\mathcal{L}_{0}}$. This property illustrates that all the slid points associated with $\mathbf{P}_{\mathcal{L}_{0}}$ cannot fold over each other along $\mathbf{C}_{P}$. This property combines with $(\mathrm{H})$ to ensure that every slid point $\mathbf{P}^{\prime}$ preserves the same neighbors as those of $\mathbf{P}$.

Once the sliding of each vertex $\mathbf{P} \in \mathcal{S}_{b}$ is computed, we obtain the retracted surface mesh $\mathcal{S}_{b}^{\prime}$ whose boundary is as close as possible to $\mathcal{L}_{t}$ to ensure a tension loading during its deformation process under ASAN.

Advantages of the retraction The computation of each $\mathbf{u}_{P}$ is no longer order-dependent: it can be performed in a single loop for every $\mathbf{P} \in \mathcal{S}_{b}$. Moreover, if the initial mesh presents symmetry properties and $\mathcal{L}_{0}$ and $\mathcal{L}_{t}$ follow these properties as well, then the retraction preserves these properties (criterion $c_{3}$ ) as shown in Figure 12(c). Thus, it satisfies all three criteria. The retraction process produces a boundary $\mathcal{L}_{0}^{\prime}$ that is smoother than that of the sequential sliding (see Figure $11(\mathrm{c})), \mathcal{L}_{0}^{\prime}$ stays close to $\mathcal{L}_{t}$ and can avoid the occurrence of wiggles in $\widetilde{\mathcal{S}}_{b}$. Further, the described retraction process is able to handle complex domains, possibly with internal constraints (see Figure 16).

In the remaining of our work, the retraction of $\mathcal{S}_{b}$ into $\mathcal{S}_{b}^{\prime}$ is thus used as a pre-processing step in our deformation pipeline, defined for the configurations where the $\mathcal{L}_{t}$ is on-surface.

\section{ASAN for Feature Lines (ASAN-FL)}

In the previous section, the retraction process has been described in details. This process corresponds to the step 4 of our ASAN-FL deformation. Let us now briefly come back to the remaining steps of our ASAN-FL deformation as enumerated in Section 3.5 and illustrated in Figure 8. 
Detection of on/off-surface configurations The distinction of FL modification categories on- or off-surface (see Section 3.4) is based on loadings, which derive from the relative displacements from $\mathcal{L}_{0}$ to $\mathcal{L}_{t}$ along $\mathcal{S}$. On the one hand, such displacements may vary along $\mathcal{L}_{0}$ when $\mathcal{L}_{t}$ undulates around $\mathcal{L}_{0}$, resulting into a subset of $\mathcal{L}_{0}$ subjected to traction and another one to compression. On the other hand, the dihedral angle along $\mathcal{L}_{0}$ must be preserved as much as possible (see Section 3.5), which can be efficiently achieved with ASAN without retraction for off-surface configurations. As a result, one has to decide between the domain decomposition required for on-surface processing and the off-surface processing without such a decomposition. As a first step, we use the following heuristic to detect whether $\mathcal{L}_{t}$ is on- or off-surface.

We define $\mathcal{L}_{0}$ as off-surface if more than half of its edges undergoes a displacement $\mathbf{u}$ that can be characterized locally as off-surface as follows. Let $\mathbf{u}:=\left(\mathbf{u}_{i}+\right.$ $\left.\mathbf{u}_{i+1}\right) / 2$ be the displacement (1) of the edge $P_{i} P_{i+1} \in \mathcal{L}_{0}$ and let us use the indices $\alpha$ and $\beta$ to designate quantities on each side of $\mathcal{L}_{0}$. Then, let $u_{N_{\alpha}}=\mathbf{u}_{i} \cdot \mathbf{N}_{\alpha}$ the normal component, and $u_{T_{\alpha}}$ the in-plane component with respect to the neighboring triangle $F_{\alpha}$ of the edge $P_{i} P_{i+1}$ (analogous setting for $F_{\beta}$ ). This edge is counted as off-surface into the set $E_{\text {off }}$ if $u_{N_{\alpha}} \cdot u_{N_{\beta}}>0$ and either $\left|u_{N_{\alpha}}\right|>\left|u_{T_{\alpha}}\right|$ or $\left|u_{N_{\beta}}\right|>\left|u_{T_{\beta}}\right|$. Finally, we consider that $\mathcal{L}_{t}$ is off-surface when:

$$
\frac{l_{\text {off }}}{l_{\mathcal{L}_{0}}}>\frac{1}{2}
$$

where $\quad l_{o f f}=\sum_{j \in E_{o f f}}\left\|\mathbf{P}_{j} \mathbf{P}_{j+1}\right\| \quad$ and $\quad l_{\mathcal{L}_{0}}=$ $\sum_{i=1}^{n-1}\left\|\mathbf{P}_{i} \mathbf{P}_{i+1}\right\|$. At the opposite, if $\mathcal{L}_{0}$ is on-surface, the processing pipeline with steps 1 to 7 of our approach summarized in Section 3.5 is applied with the following details.

\subsection{Extension of $\mathcal{L}_{0}$ and (Step 1)}

As seen in Section 3.4 , the ROI $\mathcal{S}$ is likely to be subjected to different mechanical loadings on each side of $\mathcal{L}_{0}$. Thus, we need to treat each side separately. However, it happens frequently that $\mathcal{L}_{0}$ does not have any intersection with the ROI border $\mathcal{F}$. In order to clearly distinguish each side, $\mathcal{S}$ must be partitioned and, as a first step, we extend $\mathcal{L}_{0}$ up to $\mathcal{F}$. We therefore define, at each extremity $\mathbf{P}$ of $\mathcal{L}_{0}$, the normal plane $\Pi$ containing the tangent to $\mathcal{L}_{0}$ at $\mathbf{P}$ and the normal vector to $\mathcal{S}$. We then compute the intersection point $\mathbf{Q}$ between $\Pi$ and $\mathcal{F}$. Using Dijkstra's algorithm, we find the shortest path of mesh edges in $\mathcal{S}$ that extends $\mathcal{L}_{0}$ from $\mathbf{P}$ to $\mathbf{Q}$. Extending $\mathcal{L}_{0}$ at each of its extremities defines $\widehat{\mathcal{L}_{0}}$.

\subsection{Cutting of the ROI into $\mathcal{S}_{a}$ and $\mathcal{S}_{b}$ and setting of $\mathcal{S}_{b}$ (Step 2)}

Given $\widehat{\mathcal{L}_{0}}$, we get a sequence of edges crossing $\mathcal{S}$ and intersecting $\mathcal{F}$ twice, thus defining two adjacent subdomains $\mathcal{S}_{a}$ and $\mathcal{S}_{b}$. Duplicating the vertices and edges of $\widehat{\mathcal{L}_{0}}$ enables the separation of $\mathcal{S}_{a}$ and $\mathcal{S}_{b}$ while preserving their compatibility for a subsequent merge.

To set one side of $\mathcal{L}_{0}$ as $\mathcal{S}_{b}$, i.e., the compressed side, let us consider the projections $u_{T_{\alpha}}, u_{T_{\beta}}$, previously computed for $i^{\text {th }}$ edge of $\mathcal{L}_{0}$. Let $s_{\alpha}=\sum_{j=1}^{n-1}\left|u_{T_{\alpha j}}\right|$, $s_{\beta}=\sum_{j=1}^{n-1}\left|u_{T_{\beta j}}\right|$, quantify the deviation of $\mathcal{L}_{t}$ measured along the sides $\alpha$ and $\beta$, respectively. Then, as another heuristic, $\max \left(s_{\alpha}, s_{\beta}\right)$ lets us identify the side where the compression is prominent. We note the corresponding part $\mathcal{S}_{b}$, while $\mathcal{S}_{a}$ is the part under a tension loading.

\subsection{Surface deformation (ASAN) on $\mathcal{S}_{a}$ (Step 3)}

As $\mathcal{S}_{a}$ is under traction loading, we can apply the physical-based deformation method ASAN straightforwardly. Now, the purpose is to set the ASAN deformation parameters appropriately. ASAN requires two parameters $\left(\mu, \mathbf{d}_{o}\right)$ to characterize the anisotropy of the deformation domain. $\mu$ sets the ratio of the Young's moduli and, following [30], it is assigned $\mu=100$ over the sub-domains. $\mathbf{d}_{o}$ defines the orthotropy direction. Contrary to [30], we do not have here a unique user-defined displacement of the handle, instead we use the displacements $\mathbf{u}_{i}$ computed for the vertices of $\mathcal{L}_{0}$ (see eq. (1)) to define a direction of orthotropy by $\mathbf{d}_{o}=\frac{\sum_{i} \mathbf{u}_{i}}{\left\|\sum_{i} \mathbf{u}_{i}\right\|}$. This setting completes the ASAN parameter definition on $\mathcal{S}_{a}$ to obtain $\widetilde{\mathcal{S}_{a}}$.

\subsection{Surface deformation (ASAN) on $\mathcal{S}_{b}^{\prime}$ (Step 5)}

After the retraction, since the vertices of $\mathcal{L}_{0}$ have slid, they are part of the new polyline $\mathcal{L}_{0}^{\prime} . \mathcal{L}_{0}^{\prime}$ still remains on the surface $\mathcal{S}_{b}$ and must be mapped onto $\widehat{\mathcal{L}}_{t}$. To do so, we compute new displacements $\mathbf{u}_{i}^{\prime}:=\mathbf{P}_{i}^{t}-\mathbf{P}_{i}^{\prime}$ corresponding to weights $\lambda=1$, as described in Section 4.3. 


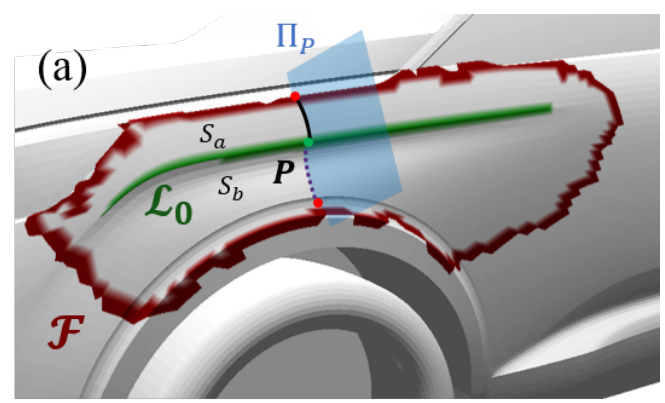

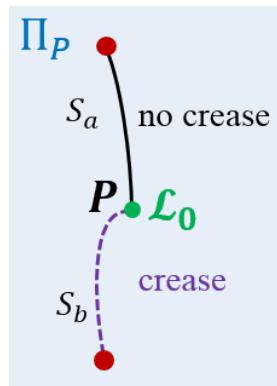

(b)

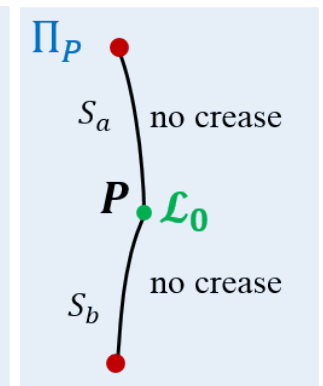

(c)
Figure 15: (a) $\Pi_{P}$ is the plane normal to $\mathcal{L}_{0}$ at a point $\mathbf{P}$ (b) Intersection curves of $\mathcal{S}_{a}$ and $\mathcal{S}_{b}$ in $\Pi_{P}$. $\mathcal{S}_{a}$ has a slowly varying curvature near $\mathcal{L}_{0}$ and has no crease while $\mathcal{S}_{b}$ has a rapidly varying curvature creating a crease. (c) A setup where the visual sharpness has been lost.

The anisotropy direction $\mathbf{d}_{o}$ is then defined according to $\mathbf{u}_{i}^{\prime}$ by $\mathbf{d}_{o}=\frac{\sum_{i} \mathbf{u}_{i}^{\prime}}{\left\|\sum_{i} \mathbf{u}_{i}^{\prime \prime}\right\|}$.

\subsection{Merging of the deformation results (Step 6)}

Once $\mathcal{S}_{a}$ and $\mathcal{S}_{b}^{\prime}$ have been deformed, we need to "sew" their results $\widetilde{\mathcal{S}_{a}}$ and $\widetilde{\mathcal{S}_{b}}$ to recover the initial topology of $\mathcal{S}$. Since we initially duplicated the vertices along $\widehat{\mathcal{L}_{0}}$ to obtain $\mathcal{S}_{a}$ and $\mathcal{S}_{b}$, and that we prescribed compatible target locations as described at Section 5.3, we guarantee a $\mathcal{C}^{0}$ continuity of the resulting surface $\widetilde{\mathcal{S}}$ across $\mathcal{L}_{t}$. Given that $\mathcal{L}_{0}$ was initially defined as a sharp line with $\mathcal{C}^{0}$ continuity, we do not seek for a higher continuity once $\widetilde{\mathcal{S}_{a}}$ and $\widetilde{\mathcal{S}_{b}}$ are merged within our design review context.

\subsection{Normal correction along $\mathcal{L}_{t}($ Step 7)}

As a result of the deformation process, the visual sharpness of the TL may attenuate (see Figure 21). Where this happens, its visual aspect perceived by the user (e.g. a designer) as a modification of shades along $\mathcal{L}_{t}$, is directly linked to the dihedral angle initially associated with each edge along $\mathcal{L}_{0}$. This crease, due to a rapid curvature variation on one side, must be considered as a distinctive feature of the FL (see Figures 15(a), (b)). Preserving it increases the quality of the deformed surface $\widetilde{\mathcal{S}}$.

To this end, let us consider the case where the crease is located on $\mathcal{S}_{b}$ (see Figure 15). Then, its deformation, and more particularly the retraction step, can strongly modify the dihedral angle along $\widehat{\mathcal{L}_{0}}$. The 'material removal' principle of the retraction can indeed wipe out the crease from $\mathcal{S}_{b}^{\prime}$. For example, if the dihedral angle becomes too small in $\widetilde{\mathcal{S}}$, the TL will disappear as $\widetilde{\mathcal{S}}$ will appear smooth. Such a configuration requires an additional deformation step in order to transfer the initial dihedral angle along $\mathcal{L}_{0}$ onto $\mathcal{L}_{t}$.

This complementary deformation is ASAN-based and applied to $\widetilde{\mathcal{S}_{b}}$ by adding the following boundary conditions. For each edge along $\mathcal{L}_{t}$, we keep fix its neighboring triangular face belonging to $\mathcal{S}_{a}$ and apply a rigid body rotation to the its adjacent face located into $\widetilde{\mathcal{S}}_{b}^{\prime}$, in order to restore the initial dihedral angle at this edge. Then, we apply an ASAN deformation to $\widetilde{\mathcal{S}}_{b}^{\prime}$ by taking the vertices of the rotated faces as boundary condition. A similar technique was used in [7] in order to prescribe $C^{1}$ continuity across patch boundaries. The resulting deformation enables the recovery of the visual aspect of the initial FL (see Figure 21 for an application). A complementary classification of feature lines is given in Appendix A.

\section{Results}

The results illustrate various examples of FL modifications on an industrial car body and on a well known mechanical part, the fandisk. All examples focus on on-surface configurations since off-surface ones have already been illustrated in Section 3.4 with Figure 6 .

A detailed comparison with state-of-the-art methods will be presented. Among all membrane-like deformation methods, we have implemented 3 methods known for exhibiting a stiffening effect $[8,9,30]$, a useful 
property when dealing with man-made shapes. Let us give a quick reminder of the chosen methods. ARAPs\&r [8] sets a reference behavior of a membrane-like non linear mechanical model taking into account finite rotations. ASAN [30] is based on ARAP-s\&r with an additional anisotropic material to produce directional stiffness. ARAP- $n$ [9], an extension of ARAP-s\&r, enables the comparison with ASAN-FL. The extension is based on the vertex neighborhood used to compute local rotations and acts as a means to stiffen the structure. Here, $n$ characterizes the $n$-neighborhood of a vertex used to compute local rotations and strain energy. Also, LRI [32] is used for comparison with a geometric deformation approach that handles finite rotations, sharing similarities with the non linear mechanical model and extended to take into account a line modification.

The results have been obtained using a $\mathrm{C}++$ application, based on LibIGL [25] open source library and the appropriate incorporation of the aforementioned deformation methods.

\subsection{Analysis and validation}

Analysis of the final shape $\widetilde{\mathcal{S}}$ through various methods Let us start by showing the robustness and efficiency of the sliding mechanism with large displacements between $\mathcal{L}_{0}$ and $\mathcal{L}_{t}$ in Figures 16, 19 and 20.

The first example in Figure 16 simulates the tapebased modification of designers as illustrated in Figure 1. To this end, $\mathcal{L}_{t}$ is defined as a spline curve lying on $\mathcal{S}$ so that it matches precisely the configuration on-surface and follows the criteria of Section 5. Figure 16(a) shows the input domain, the ROI $\mathcal{F}$ drawn by the user (red) and the FL selected (green) together with the TL (blue). The top row depicts shaded surfaces of $\mathcal{S}$ and $\widetilde{\mathcal{S}}$. The bottom row shows maps of discrete Gaussian curvature sign of the corresponding meshes. Figure 16(a)-bottom serves as reference.

The result of ARAP-s\&r in Figure 16(b) highlights a lack of stiffness and significant curvature sign changes.

The stiffening effect of the orthotropic material defined in ASAN appears in Figure 16(c) but the bulging effect is clearly present (left part of $\mathcal{L}_{t}$ ). This is validating the need for the retraction process.

ARAP- $n$ in Figure 16(d) is applied globally over $\mathcal{S}$ without retraction. It requires a large vertex neighborhood $n$ to obtain a stiffness effect global enough. $\widetilde{\mathcal{S}}$ looks quite satisfactory. However, a close up near $\mathcal{L}_{t}$ (see Figure 17) shows that increasing $n$ does not:
- completely remove the bulging effect near $\mathcal{L}_{t}$ but merely shrinks it. This is highlighted in Figure 17(a) where a clipping plane has been used to analyze $\widetilde{\mathcal{S}}$ and the corresponding clipping polyline exhibits a small edge near $\mathcal{L}_{t}$. This is characteristic of a bulging effect squashed along $\mathcal{L}_{t}$ and this small edge belongs to a triangle squashed along $\mathcal{L}_{t}$;

- produce a satisfactory discrete Gaussian curvature sign map. The bulged and squashed area analyzed in Figure 17(a) generates a small sharp ridge that is slightly visible in Figure 17(b), (area A). Additionally, small undulations appear near $\mathcal{F}$, along the symmetry plane (area B) as highlighted when looking at the sign map of the entire hood.

Finally, ASAN-FL in Figure 16(e) illustrates the efficiency of the retraction process, removing the surface bulging and preserving the Gaussian curvature sign distribution as expected everywhere within the ROI. Further, mesh triangles in $\widetilde{\mathcal{S}}_{b}$ are not distorted under the retraction and deformation processes, which confirms the quality of the shape produced by ASAN-FL. There is no crease on $\mathcal{S}_{b}$, so that no normal correction is required.

The second example in Figure 18 is a variant of the previous configuration, where $\mathcal{L}_{t}$ is more regular and slightly above the surface $\mathcal{S}$. It is still identified as an on-surface configuration that exhibits a bulging phenomenon under ASAN deformation. Also, ARAP- $n$ contains similar artefacts as those observed in the previous case, particularly visible at the top extremity of $\mathcal{L}_{t}$. ASAN-FL performs correctly again in this case.

Results on a mechanical part Figure 19 shows another comparison between ARAP- $n$ and ASAN-FL. Here again there is no crease on $\mathcal{S}_{b}$, thus no normal correction is needed, which enables a precise comparison of the results. Both methods give similar global results (see Fig. 19(b) and (d)) and, at first sight, both visually satisfying. However, with ARAP- $n$, a high value of $n$ is needed to minimize surface bulging, which leads to degenerated triangles along $\mathcal{L}_{t}$ when taking a closer look at this area (Fig. 19(c)). Indeed, one can see that the compression taking place in $\mathcal{S}_{b}$ forms a bump, or a fold, near $\mathcal{L}_{t}$, which is not acceptable and confirms the observation made in Figure 17. Such a behavior can be correlated with the bulging effect observed near the handle $\mathcal{H}$ in examples of Le Vaou et al. [30] that is inherent to ARAP- $n$ with large neighborhoods. 


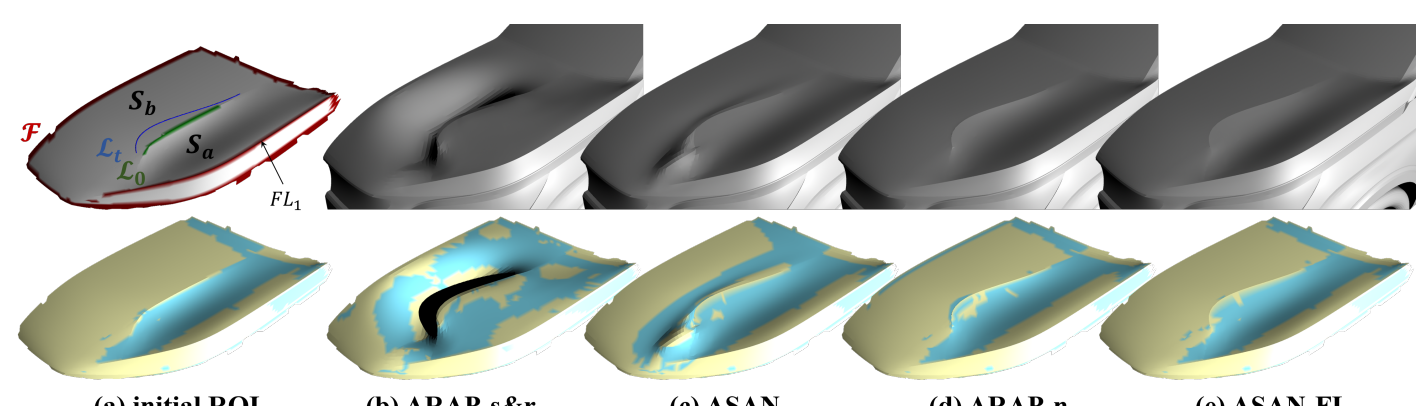

(a) initial ROI

(b) ARAP-s\&r

(c) ASAN

(d) ARAP-n

(e) ASAN-FL

Figure 16: FL modification on the hood of a car body. Top row: shaded surfaces of $\widetilde{\mathcal{S}}(\mathrm{b}-\mathrm{e})$, bottom row: maps of discrete Gaussian curvature sign.
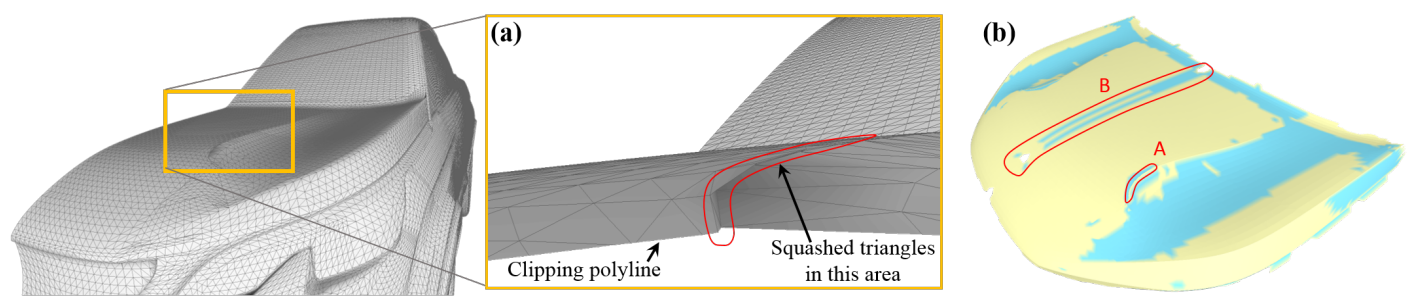

Figure 17: ARAP- $n$ results detailed analysis. (a) Close up along the ridge of the TL showing the existence of squashed triangles along $\mathcal{L}_{t}$ as part of a bulging effect that is minimized but not removed. (b) Discrete Gaussian curvature sign map showing small undulations close to the symmetry plane of the hood.

Thanks to the retraction step, our method behaves better (Fig. 19(d)) and yields a more homogeneous deformation along $\mathcal{L}_{t}$ (Fig. 19(e)), with a progressive deformation of the triangle shapes in $\mathcal{S}_{b}$ near $\mathcal{L}_{t}$. Also, Figure 19(e) shows that ASAN-FL preserves well the flatness of $\mathcal{S}_{b}$, as depicted by the orthogonal sections (thick parallel polylines). The result of ASAN-FL is thus satisfying, and shows the interest of relying on a mechanical behavior that can be analyzed and understood.

Additionally, note that the flatness of $\mathcal{S}_{b}$ is preserved by $\widetilde{\mathcal{S}}_{b}$ without requiring additional constraints contrary to $[44,10]$. Indeed, the orthotropy direction $\mathbf{d}_{o}$ and the large value of $\mu$ (related to the Young's moduli) provided by ASAN, (see step 3 in Sect. 5.3), yield this shape preserving behavior.

Retraction efficiency In Figures 20 and 21, we examine in more details the efficiency of the retraction method using another industrial example. The FL is particularly challenging to modify because of its proximity to the wheel arch and the narrow elongated shape of the ROI. Also, $\mathcal{S}_{b}$ presents a crease along $\mathcal{L}_{0}$ highlighted in Figure 21(a) and normal correction is required to restore the shaded area under the TL. This correction is solely applied to ASAN-FL with retraction (see Figure 20(h)).

ARAP-s\&r result is shown in Figure 20(b) as reference for membrane-like deformation behaviour. LRI in Figure 20(c) behaves better than ARAP-s\&r in the sense that it reduces significantly the surface bulging, taking advantage of its ability to handle finite rotations. But undulations remain (circled area) and there is no physical interpretation to be used to modify LRI and try to remove them. ARAP- $n$ results in Figure 20(d) are produced with a value of $n$ high enough to stiffen the surface. Note that the chosen value $(n=40)$ covers the whole ROI: increasing this value would not improve the deformation behavior. However, even with this maximal possible value, artefacts remain at the extremity of $\mathcal{L}_{t}$ and near the boundary of the ROI, as circled on Figure 20(d).

Then, variants of ASAN are used in three configurations (see Figures 20(f,g,h)). Figure 20(f) shows that the anisotropic stiffening effect of ASAN alone does 


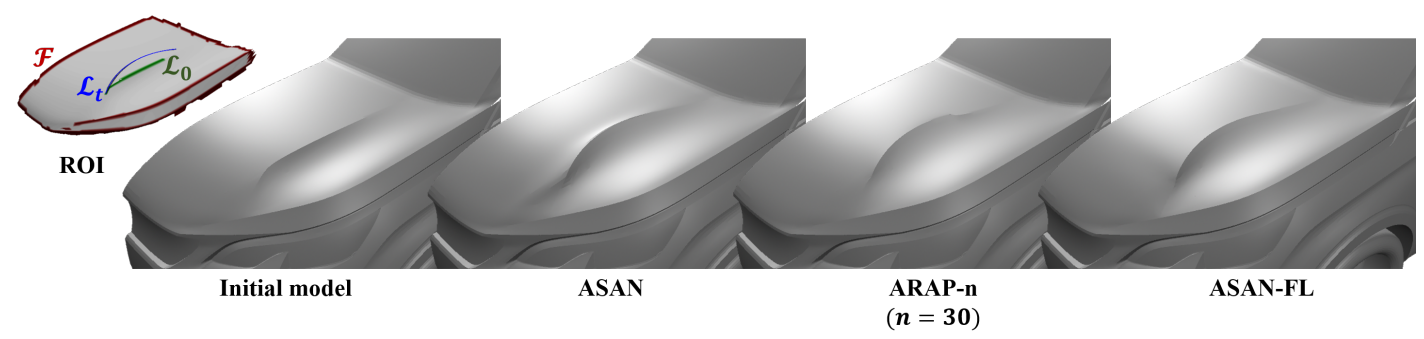

Figure 18: FL modification on the hood of the car model with a variant of $\mathcal{L}_{t}$ of Figure 16.

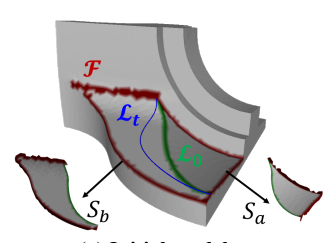

(a) Initial model

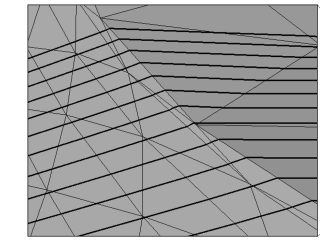

(c) ARAP-n with sections orthogonal to user-defined direction

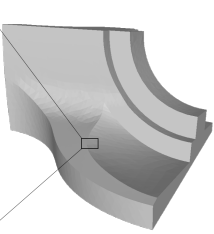

(b) ARAP-n $(n=20)$

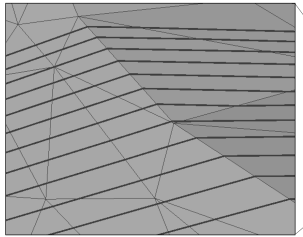

(e) ASAN-FL with sections orthogonal to user-defined direction

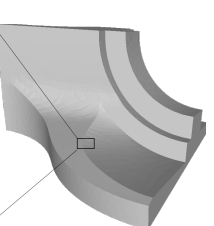

(d) ASAN-FL

Figure 19: FL modification on the fandisk model. (a) Initial model and constraints applied to the ROI: $\mathcal{L}_{0}, \mathcal{L}_{t}$ and $\mathcal{F}$. If the result of ARAP- $n$ is visually satisfying (b), this method produces degenerated triangles along $\mathcal{L}_{t}$, as shown in (c), thus creating a slight bump. This is shown where the sections (parallel thick lines) are broken near the TL. For ASAN-FL, we separate $\mathcal{S}_{a}$ (in tension) from $\mathcal{S}_{b}$ (in compression) and apply the retraction on $\mathcal{S}_{b}$. The result of ASAN-FL (d) is visually satisfying, and preserves the flatness of the surface, as shown in (e) where the sections are straight, as well as the quality of the mesh.

indeed reduce but not remove bulging, as explained at Section 3.4. The sequential sliding, used as a preprocessing step (see Sect. 4.2 and Fig. 11), does not give a satisfying result either. The too large sliding amplitude around the extremities of $\mathcal{L}_{0}^{\prime}$ and the lack of smoothness of $\mathcal{L}_{0}^{\prime}$ causes indeed mechanical plies where the surface is stretched, as indicated on Figure $20(\mathrm{~g})$ with circled area and the line indicating the ply direction. However, this behavior indicates a physical phenomenon that can be interpreted and analyzed to be removed with a smoother boundary of $\mathcal{S}_{b}^{\prime}$.

Finally, when using the retraction (with simultaneous vertex sliding), the boundary $\mathcal{L}_{0}^{\prime}$ of $\mathcal{S}_{b}^{\prime}$ becomes smoother (see Figure 11(c)) and gets closer to $\mathcal{L}_{t}$. This setting reduces the stretching of $\mathcal{S}_{b}^{\prime}$ to $\widetilde{\mathcal{S}}_{b}^{\prime}$ and homogenizes the strains in $\widetilde{\mathcal{S}}_{b}$ up to the removal of the plies, which produces a satisfactory result shown in Figure 20(h).

Preservation of the FL's visual aspect The normal correction process is illustrated in Figure 21 with an example where $\mathcal{S}_{b}$ contains a crease near $\mathcal{L}_{0}$. Without normal correction, one observes in Figure 21(b) that the visual perception of $\mathcal{L}_{0}$ fades away along $\mathcal{L}_{t}$. This phenomenon is directly deriving from the principle of material removal of the retraction, because the highly curved area near existing in $\mathcal{S}_{b}$ near $\mathcal{L}_{0}$ may vanish in $\mathcal{S}_{b}^{\prime}$ near $\mathcal{L}_{0}^{\prime}$, thus calling for the correction process.

\subsection{Performances}

The goal was to achieve interactive performance rates, allowing the use of our algorithm in an immersive system. We set the computation time of the ASAN method as a reference to better see the impact of the ROI cutting and the computation of the retraction step. The computation times were obtained on an Intel(R) Core(TM) i5-4210U @1.70GHz.

We compare in Figure 22 the computation time of ASAN (dark blue) and of ASAN-FL. The results are ordered from smallest to largest ROI sizes, detailed in the figure caption. Regarding ASAN-FL, we divided the time dedicated to the computation of the deformation (light blue) and the retraction (orange). Since in ASAN-FL, the deformation is computed on each part $\left(\mathcal{S}_{a}\right.$ and $\mathcal{S}_{b}$ both smaller than the ROI), the deformation steps are quicker and the overall time dedicated to the 


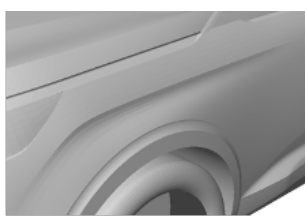

(a) Initial model

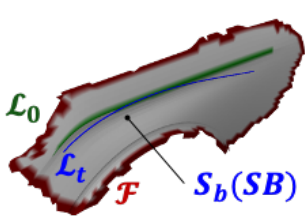

(e) ROI

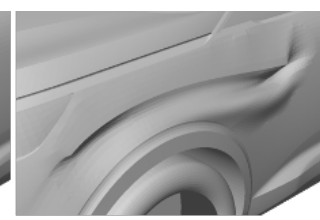

(b) ARAP-s\&r

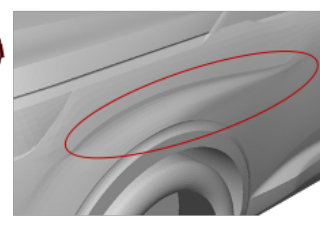

(f) ASAN

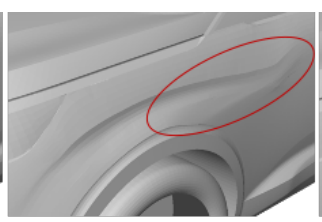

(c) LRI

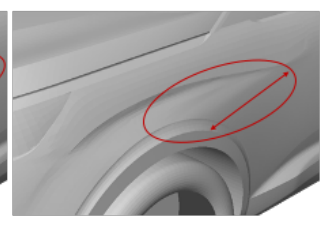

(g) ASAN-FL

(sequential sliding)

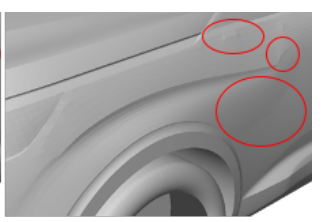

(d) ARAP-n

$(n=40)$

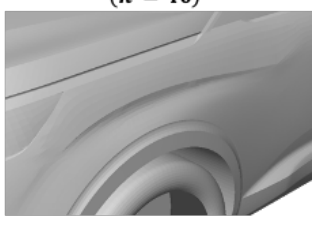

(h) ASAN-FL

(retraction)

with normal correction

Figure 20: Modification of a FL above the wheel arch. (a) Initial model and (e) ROI with constraints $\mathcal{L}_{0}, \mathcal{F}$ and $\mathcal{L}_{t}$. Results with: ARAP-s\&r (b), LRI (c), ARAP- $n$ (d), ASAN (f), ASAN-FL with sequential sliding (g) (here, $\mathcal{L}_{0}^{\prime}$ is not smooth enough near its extremities (cf Fig. 11(b)), which creates plies that are not acceptable), ASAN-FL with retraction and normal correction (h) (sliding is more homogeneous (cf. Fig. 11(c)), plies disappear, producing a satisfactory result).

deformation is less than for the ASAN deformation.

The retraction time stays in the same order of magnitude but seems to increase with the size of the ROI. Figure 23 shows that the retraction time evolves quadratically wrt the number of vertices.

\section{Discussion}

The results demonstrate the prominence of aesthetic issues on the evaluation of shape quality. If the maps of Gaussian discrete curvature (see Figure 16) has appeared as a valuable evaluation criterion for the comparison of deformation methods, it could serve as well for deriving a criterion for an automated detection of crease bends requiring normal correction along the resulting feature line. For now, and because it is coherent in a modification (not a creation) context, similar shapes were chosen for $\mathcal{L}_{t}$ and $\mathcal{L}_{0}$ in our examples. However, since the physical behavior of the ASAN model is predictable, the method could be adapted to handle more complex configurations. For example, cases with $\mathcal{L}_{t}$ 'zigzagging' around $\mathcal{L}_{0}$ could be handled with the application of the retraction process on both sides of $\mathcal{L}_{0}$. Further, large shape differences between $\mathcal{L}_{0}$ and $\mathcal{L}_{t}$ could create more bulging phenomena along different directions, which is calling for further developments of the proposed approach.

It may happen that $\widetilde{\mathcal{S}}$ lacks smoothness at the extremities of $\mathcal{L}_{t}$ when normal correction is applied. $\mathcal{L}_{t}$ is user-defined and must be regarded as an approximation of the desirable solution, as pointed out by Cheutet et al. [10], rather than a hard constraint. Consequently, the extremity of the feature line on the resulting surface, which is a cone singularity, should slide along $\mathcal{L}_{t}$ to generate a smoother surface whenever possible. This aspect is related to the continuity across the resulting surface parts $\widetilde{\mathcal{S}_{a}}$ and $\widetilde{\mathcal{S}_{b}}$ along the extension of $\mathcal{L}_{t}$. Raising the $C^{0}$ continuity here is left for another future contribution.

Also, this improvement on continuity conditions could help unify the processing of on/off configurations. Currently, applying the surface decomposition and the retraction on off-surface configurations is not relevant, because they are processed faster and their deformed domain is intrinsically smoother than onsurface ones. Preserving such smoothness after domain decomposition, and combining it with the improvement described in the above paragraph, requires further contribution. This would lead however, to the unification of on/off configurations and enable the removal of the heuristic that currently distinguishes them.

Similarly, functional constraints could be added to monitor some shape features of $\mathcal{S}$, such as preservation of planar or cylindrical areas [10, 44], which are of importance for man-made objects. However, it has been observed that ASAN-FL is already giving some equivalence with functional constraints setting a fixed area, planar areas, or preserving a symmetry plane (when the 


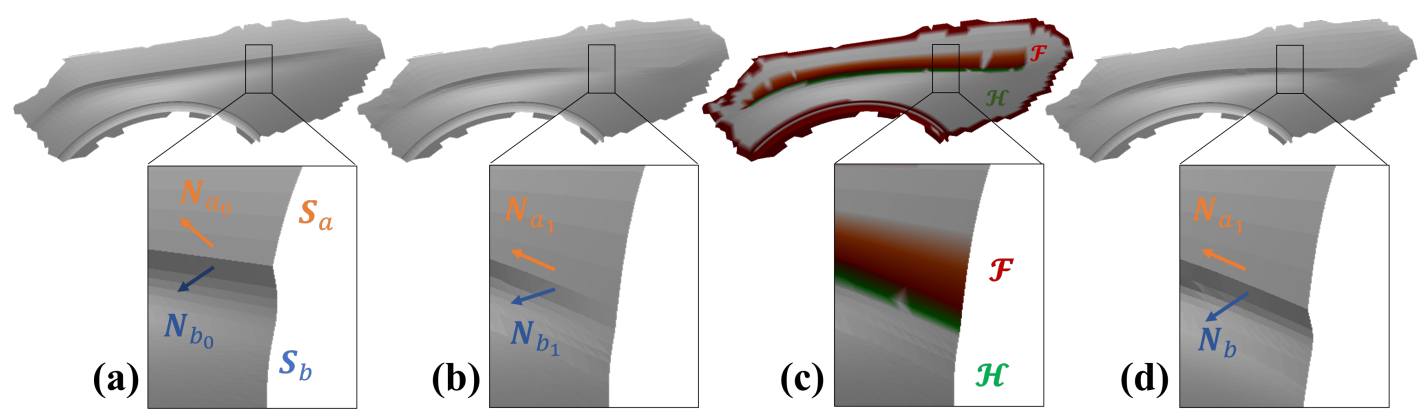

Figure 21: Normal correction process. For each sub-figure, a 3D view is displayed on the top and an annotated close-up, with vertical section, at the bottom. (a) Initial ROI. (b) Mesh after deforming $\left(\mathcal{S}_{a}, \mathcal{S}_{b}^{\prime}\right)$ and merging $\left(\widetilde{\mathcal{S}}_{a}, \widetilde{\mathcal{S}}_{b}^{\prime}\right)$. The initial dihedral angle is modified: the visual aspect of $\mathcal{L}_{t}$ misses that of $\mathcal{L}_{0}$. (c) To recover it, we apply a deformation that constrains all vertices of triangles sharing an edge with $\mathcal{L}_{t}$. The vertices belonging to $\mathcal{S}_{a}$ and $\mathcal{L}_{t}$ are fixed (belong to $\mathcal{F}$ ) while a rotation is set on vertices of $\mathcal{S}_{b}^{\prime}$ (in $\mathcal{H}$ (see Section 5.6)). (d) This deformation lets us recover the visual aspect of $\mathcal{L}_{0}$.

anisotropy direction is orthogonal to the targeted symmetry plane). This is also an advantage of ASAN-FL that incorporates appropriate structural stiffness to express shape features without requiring complementary computations, compared to the overhead brought by methods requiring these constraints explicitly.

\section{Conclusion}

The FL modification process, as performed in the context of the automotive industry is a challenging task since the standard physically-based approaches exhibit predictive undesirable effects such as surface bulging. We analyzed this process by considering the surface deformation as a response to a mechanical tension or compression loading. The distinction of feature lines in two categories, on and off-surface, enabled us to propose a shape retraction process in the ROI sub-domain under compression loading so that the resulting surfaces behaves as expected without bulging or generating plies. Further, the retraction process prevents the surface deformation from generating plies, predictably appearing when high tension ratio appear, and preserves the intrinsic symmetry properties in the ROI, when they exist.

The comparison of the proposed ASAN-FL deformation process, which is a physically-based deformation method close to a non linear membrane-like mechanical model with an anisotropic stiffening behavior, has been validated through test cases based on industrial car bodies and comparison with state-of-art deformation methods.
ASAN-FL turns out to be an efficient and robust deformation method with predictive physical behavior, that can be extended and adapted to other domains of interest than car body design.

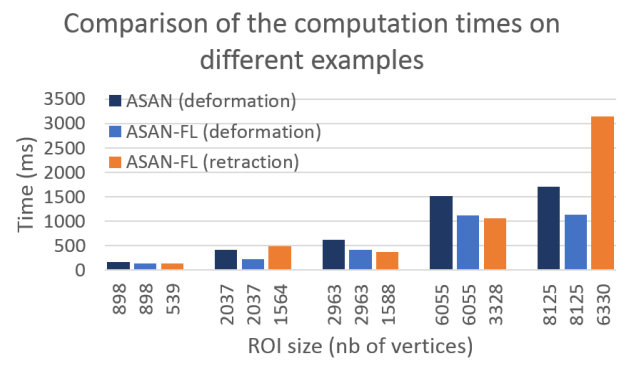

Figure 22: For each example, comparison between the computation times of ASAN deformation (left columns, dark blue) and those of our ASAN-FL method, decomposed into deformation (light blue) and retraction (orange) steps.

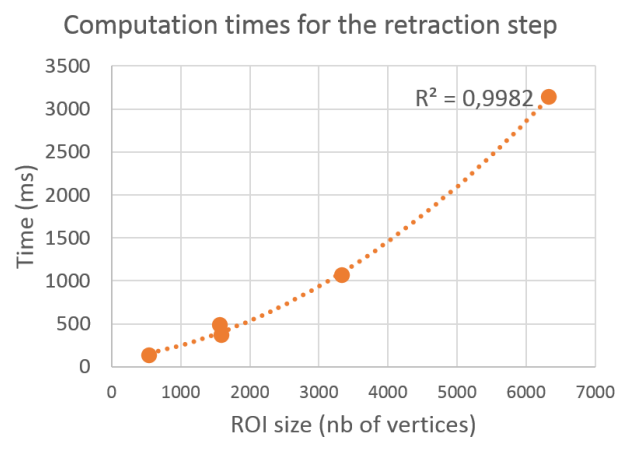

Figure 23: Fitting of average computation times for the retraction step (see Figure 22) highlighting a quadratic behavior. 
Acknowledgments The fandisk model is provided courtesy of MPII, by the AIM@SHAPE Repository. The car models are courtesy of Groupe PSA. This work was partially funded by the ANRT contract $n^{\circ} 2016 / 1107$. We thank PiXYZ [3] who helped us converting the car models into meshes.

\section{A Complementary classification of Feature Lines}

In addition to the feature line classification on and offsurface, the sharpness of the feature line stemming from a more or less sharp curvature bend or crease on either side of $\mathcal{L}_{0}$ calls for further distinctions.

The existence of areas with sharp bends $(S B)$ in contrast to areas with slowly varying curvatures, i.e., no bend $(N B)$, along $\mathcal{L}_{0}$ is a geometric feature independent of the traction/compression loading concept used to avoid bulging areas. Further, the context of car body design highlights $\mathcal{L}_{0}$ with neighborhoods of type $(S B, N B)$ (see $\mathrm{FL}_{1}$ on Figure $16(\mathrm{a})$ ) and $(N B, N B)$ (see Figure 15(c)), only. It is open to consider that FL of type $(S B, S B)$ can be of interest for other contexts but the focus stays here on car body design and $(S B, S B)$ is not part of the classification. Then, $(N B, N B)$ has not appeared as requiring some normal correction since the effect of the retraction process, if needed, does not significantly modify the curvature distribution along $\mathcal{L}_{0}^{\prime} \in \mathcal{S}_{b}^{\prime}$.

In terms of taxonomy, this leaves the configurations derived from $(S B, N B)$ combined with $\left(\mathcal{S}_{a}, \mathcal{S}_{b}^{\prime}\right)$ if $\mathcal{L}_{t}$ is on-surface since off-surface configurations haven't highlighted any significant alteration of the dihedral angle through the surface deformation process. As a result, two combinations occur when $\mathcal{L}_{t}$ is on-surface:

- $S B$ lies inside $\mathcal{S}_{a}$ (see Figure 16) and the retraction process has no effect on $S B$. The resulting surface $\widetilde{\mathcal{S}}$ does not exhibit a significant alteration of the dihedral angle, hence no normal correction is required in this case;

- $S B$ lies inside $\mathcal{S}_{b}$ (see Figures 20 and 21). This is the configuration described in section 5.6.

\section{References}

[1] https://www.gravitysketch.com/.

[2] https://www.3ds.com/ products-services/catia/products/
v5/portfolio/domain/Shape_Design_ Styling/product/IMA/.

[3] https://www.pixyz-software.com/.

[4] R. Arora, R. H. Kazi, F. Anderson, T. Grossman, K. Singh, and G. Fitzmaurice. Experimental evaluation of sketching on surfaces in VR. In Proceedings of the 2017 CHI Conference on Human Factors in Computing Systems, CHI'17, page 5643-5654, 2017.

[5] E. L. Axelrad. On local buckling of thin shells. Int. J. Non-Linear Mechanics, 20(4):249-259, 1985.

[6] R. Balakrishnan, G. Fitzmaurice, G. Kurtenbach, and W. Buxton. Digital tape drawing. In Proceedings of the 12th Annual ACM Symposium on User Interface Software and Technology, UIST'99, page 161-169, 1999.

[7] M. Botsch and L. Kobbelt. An intuitive framework for real-time freeform modeling. ACM Trans. Graph., 23(3):630-634, Aug. 2004.

[8] I. Chao, U. Pinkall, P. Sanan, and P. Schröder. A simple geometric model for elastic deformations. ACM Trans. Graph., 29(4):38:1-38:6, July 2010.

[9] S.-Y. Chen, L. Gao, Y.-K. Lai, and S. Xia. Rigidity controllable as-rigid-as-possible shape deformation. Graphical Models, 91:13 - 21, 2017.

[10] V. Cheutet, C. E. Catalano, J. P. Pernot, B. Falcidieno, F. Giannini, and J. C. Leon. 3d sketching for aesthetic design using fully free-form deformation features. Comput. Graph., 29(6):916-930, Dec. 2005.

[11] M. Colaianni, C. Siegl, J. Süßmuth, F. Bauer, and G. Greiner. Anisotropic deformation for local shape control. Computational Visual Media, 3(4):305-313, Dec 2017.

[12] E. Dekkers, L. Kobbelt, R. Pawlicki, and R. C. Smith. A sketching interface for feature curve recovery of free-form surfaces. In 2009 SIAM/ACM Joint Conference on Geometric and Physical Modeling, SPM '09, page 235-245, 2009.

[13] É. Demers, C. Tribes, and F. Guibault. A selective eraser of curvature extrema for b-spline curves. Computers \& Graphics, 51:35-42, 2015.

[14] M. Eigensatz and M. Pauly. Positional, metric, and curvature control for constraint-based surface deformation. Computer Graphics Forum, 28(2):551-558, 2009.

[15] M. Eigensatz, R. W. Sumner, and M. Pauly. Curvature-domain shape processing. Computer Graphics Forum, 27(2):241-250, 2008.

[16] G. Farin, G. Rein, N. Sapidis, and A. Worsey. Fair- 
ing cubic b-spline curves. Computer Aided Geometric Design, 4(1):91 - 103, 1987. Topics in CAGD.

[17] M. Fiorentino, G. Monno, P. A. Renzulli, and A. E. Uva. 3d sketch stroke segmentation and fitting in virtual reality. In Proc. Intern. Conf. Computer Graphics and Vision (GRAPHICON '03), pages 188-191, 2003.

[18] R. Gal, O. Sorkine, N. J. Mitra, and D. CohenOr. iwires: An analyze-and-edit approach to shape manipulation. ACM Trans. Graph., 28(3):33:133:10, July 2009.

[19] L. Gao, C. Zhang, L. Zhu, S. Li, and G. Wang. Interactive modeling and editing of free-form surfaces from curve networks. In Proceedings of the 15th ACM SIGGRAPH Conference on VirtualReality Continuum and Its Applications in Industry - Volume 1, VRCAI '16, page 339-344, 2016.

[20] C. Grimm and P. Joshi. Just drawit: A 3d sketching system. In Proceedings of the International Symposium on Sketch-Based Interfaces and Modeling, SBIM'12, page 121-130. Eurographics Association, 2012.

[21] T. Grossman, R. Balakrishnan, G. Kurtenbach, G. Fitzmaurice, A. Khan, and B. Buxton. Creating principal $3 \mathrm{~d}$ curves with digital tape drawing. In Proceedings of the SIGCHI Conference on $\mathrm{Hu}$ man Factors in Computing Systems, CHI'02, page 121-128, 2002.

[22] K. Hildebrandt, K. Polthier, and M. Wardetzky. Smooth feature lines on surface meshes. In Proceedings of the Third Eurographics Symposium on Geometry Processing, SGP'05, page 85-es, Goslar, DEU, 2005. Eurographics Association.

[23] T. Igarashi, S. Matsuoka, and H. Tanaka. Teddy: A sketching interface for $3 \mathrm{~d}$ freeform design. In Proceedings of the 26th Annual Conference on Computer Graphics and Interactive Techniques, SIGGRAPH'99, page 409-416, USA, 1999.

[24] B. Jackson and D. F. Keefe. Lift-off: Using reference imagery and freehand sketching to create $3 \mathrm{~d}$ models in vr. IEEE Transactions on Visualization and Computer Graphics, 22(4):1442-1451, 2016.

[25] A. Jacobson, D. Panozzo, et al. libigl: A simple C++ geometry processing library, 2018. http://libigl.github.io/libigl/.

[26] D. Keefe, R. Zeleznik, and D. Laidlaw. Drawing on air: Input techniques for controlled $3 \mathrm{~d}$ line illustration. IEEE Transactions on Visualization \& Computer Graphics, 13(05):1067-1081, 2007.
[27] D. F. Keefe, D. A. Feliz, T. Moscovich, D. H. Laidlaw, and J. J. LaViola. Cavepainting: A fully immersive $3 \mathrm{~d}$ artistic medium and interactive experience. In Proceedings of the 2001 Symposium on Interactive 3D Graphics, I3D'01, page 85-93, 2001.

[28] I. Kókai, J. Finger, R. C. Smith, R. Pawlicki, and T. Vetter. Example-based conceptual styling framework for automotive shapes. In Proceedings of the 4th Eurographics workshop on Sketchbased interfaces and modeling, pages 37-44, 2007.

[29] F. Lazarus, S. Coquillart, and P. Jancène. Axial deformations: an intuitive deformation technique. Computer-Aided Design, 26(8):607 - 613, 1994. Special Issue Modelling in computer graphics.

[30] Y. Le Vaou, J.-C. Léon, S. Hahmann, S. Masfrand, and M. Mika. As-Stiff-As-Needed Surface Deformation Combining ARAP Energy with an Anisotropic Material. Computer-Aided Design, 121:1-15, Apr. 2020.

[31] Z. Levi and G. Gotsman. Smooth rotation enhanced as-rigid-as-possible mesh animation. IEE TVCG, 21(2):264:1-277:15, Feb. 2015.

[32] Y. Lipman, O. Sorkine, D. Levin, and D. CohenOr. Linear rotation-invariant coordinates for meshes. ACM Trans. Graph., 24(3):479-487, July 2005.

[33] K. Lu, M. Accorsi, and J. Leonard. Finite element analysis of membrane wrinkling. International Journal for Numerical Methods in Engineering, 50:1017-1038, 2001.

[34] Y. Luo, J. Xing, Y. Niu, M. Li, and Z. Kang. Wrinkle-free design of thin membrane structures using stress-based topology optimization. Journal of the Mechanics and Physics of Solids, 102:277293, 2017.

[35] J. McCrae and K. Singh. Sketching piecewise clothoid curves. Computers \& Graphics, 33(4):452-461, 2009.

[36] A. Nealen, T. Igarashi, O. Sorkine, and M. Alexa. Fibermesh: Designing freeform surfaces with $3 \mathrm{~d}$ curves. ACM Trans. Graph., 26(3):41-es, July 2007.

[37] G. Orbay and L. Kara. Shape design from exemplar sketches using graph-based sketch analysis. Journal of Mechanical Design, 134, 112012.

[38] H. Perkunder, J. H. Israel, and M. Alexa. Shape modeling with sketched feature lines in immersive 3d environments. In Proceedings of the Sev- 
enth Sketch-Based Interfaces and Modeling Symposium, SBIM'10, page 127-134, 2010.

[39] J. Pernot, S. Guillet, J. Léon, B. Falcidieno, and F. Giannini. Multi-minimisations for shape control of fully free form deformation features. In Proceedings of the IEEE Shape Modelling International Conference, Genova, Italy. IEEE, 2004.

[40] D. Pinskiy. Sliding Deformation: Shape Preserving Per-Vertex Displacement. In H. P. A. Lensch and S. Seipel, editors, Eurographics 2010 - Short Papers, pages 1-4, 2010.

[41] P. Santos, A. Stork, R. Filipe, and J. Jorge. An integrated approach to virtual tape drawing for automotive design. In Proceedings of HCI International, pages 1203-1207, 2005.

[42] L. Schemali, J.-M. Thiery, and T. Boubekeur. Automatic line handles for freeform deformation. In Eurographics 2012 (Short), pages 81-84, 2012.

[43] S. Schkolne, M. Pruett, and P. Schröder. Surface drawing: Creating organic $3 \mathrm{~d}$ shapes with the hand and tangible tools. In Proceedings of the SIGCHI Conference on Human Factors in Computing Systems, CHI'01, page 261-268, 2001.

[44] D. Sieger, S. Gaulik, J. Achenbach, S. Menzel, and M. Botsch. Constrained space deformation techniques for design optimization. ComputerAided Design, 72:40-51, 2016. 23rd International Meshing Roundtable Special Issue: Advances in Mesh Generation.

[45] K. Singh and E. Fiume. Wires: A geometric deformation technique. In Proceedings of the 25th Annual Conference on Computer Graphics and Interactive Techniques, SIGGRAPH'98, pages 405414. ACM, 1998.

[46] O. Sorkine and M. Alexa. As-rigid-as-possible surface modeling. In Proceedings of the Fifth Eurographics Symposium on Geometry Processing, SGP'07, pages 109-116, 2007.

[47] F. Verhoeven and O. Sorkine-Hornung. RodMesh: Two-handed 3D Surface Modeling in Virtual Reality. In H.-J. Schulz, M. Teschner, and M. Wimmer, editors, Vision, Modeling and Visualization, pages 1-10, 2019.

[48] D. Walton and D. Meek. A further generalisation of the planar cubic bézier spiral. Journal of Computational and Applied Mathematics, 236:2869-2882, 2012.

[49] C. Weber, S. Hahmann, and H. Hagen. Sharp feature detection in point clouds. In Proceedings of the 2010 Shape Modeling International Confer- ence, SMI'10, page 175-186, 2010.

[50] G. Wesche and H.-P. Seidel. Freedrawer: A freeform sketching system on the responsive workbench. In Proceedings of the ACM Symposium on Virtual Reality Software and Technology, VRST'01, page 167-174, 2001.

[51] G. Xu, K. Hui, W. Ge, and G. Wang. Direct manipulation of free-form deformation using curvepairs. Computer-Aided Design, 45(3):605 - 614, 2013.

[52] H. Zhao and S. J. Gortler. A report on shape deformation with a stretching and bending energy, 2016.

[53] H. Zhao and S. J. Gortler. Shape deformation with a stretching and bending energy. In Proceedings of the 31st Int. Conf. CASA, Beijing, China, pages 71-76, 2018.

[54] Q. Zhou, T. Weinkauf, and O. Sorkine. Featurebased mesh editing. In Proc. Eurographics, Short Papers, pages 1-4, Llandudno, UK, April 2011. 